\title{
Performance study of multiuser interference mitigation schemes for hybrid broadband multibeam satellite architectures
}

\author{
Jesús Arnau ${ }^{1 *}$, Bertrand Devillers², Carlos Mosquera ${ }^{1}$ and Ana Pérez-Neira ${ }^{2,3}$
}

\begin{abstract}
As the demand for higher throughput satellites increases, multibeam architectures with smaller beam spots are becoming common place. If the same frequency is strongly reused, the resulting interference when serving simultaneously many users requires some sort of pre or post-cancelation process. This article focuses on precoding and multiuser detection schemes for multibeam satellites, comparing hybrid on-board on-ground beamforming techniques with fully ground-based beamforming. Both techniques rely on the exchange of radiating element signals between the satellite and the corresponding gateway but, in the latter case, the interference mitigation process acts on all the radiating signals instead of the user beams directly, with the corresponding extra degrees of freedom for those cases for which the number of radiating elements is higher than the number of user beams. The analysis carried out in this study has shown that the potential advantage of ground-based beamforming may exceed $20 \%$ of the total throughput.
\end{abstract}

Keywords: multibeam satellite communication, interference mitigation, multiuser detection, precoding, hybrid architecture

\section{Introduction}

The use of multiple spot beams in modern broadband satellites has increased during the last few years in an effort to serve higher throughput demands with a scalable cost, for which frequency reuse among users is required [1]. Thus, the same frequency band is shared by different beams to provide an overall higher throughput as long as the multiuser interference can be kept under control. This interference occurs due to the non-null side lobes of the beams radiation patterns, and is related to the degree of reuse of the spectrum. A partial frequency reuse would exclude adjacent beams from using the same portion of spectrum (or color). However, more aggressive frequency reuse strategies $[2,3]$ can push forward the overall spectral efficiency provided the resulting interference can be efficiently managed. On the other side, the increase in the number of spot beams, in the desired capacity per beam, and in the frequency reuse might convert feeder

\footnotetext{
* Correspondence: suso@gts.uvigo.es

${ }^{1}$ Signal Theory and Communications Department, University of Vigo, 36310 Vigo, Spain

Full list of author information is available at the end of the article
}

links in a bottleneck. Higher frequency bands such as $\mathrm{Q} / \mathrm{V}$, optical communications or multigateway architectures need to be addressed to accommodate the required capacity.

Conventional beamforming techniques are space-based (or on-board) architectures including analog or digital beamforming networks. On-board volume and calibration requirements are perhaps their main drawbacks. In an attempt to shift the complexity to the ground segment, more recent ground-based beamforming (GBBF) techniques rely on the exchange of radiating element signals between the satellite and the gateway. The forming of beams is realized on-ground with all the flexibility offered by on-ground digital signal processing [4]. Again, at the cost of a higher feeder link bandwidth demand for those cases with more feeds or radiating elements than number of beams, more sophisticated and power consuming techniques can be implemented. Flexibility is preserved, and changes in shape, traffic and pointing direction can be accommodated. Multiuser interference mitigation schemes such as precoding or multiuser detection can be jointly designed with the beamforming process at the gateway 
station: this joint optimization process is expected to provide some gain in terms of capacity as we will show in this study. The interference mitigation process would act on all the radiating signals instead of the user beams directly, with the corresponding extra degrees of freedom.

More specifically, the forward link from the gateway to the users can be recast as a multiple-input multiple-output (MIMO) broadcast channel [5] from an information theoretic perspective, whereas the return link corresponds to the multiple access channel (MAC) [6]. In the forward link, the sum rate capacity is known to coincide with the rate region on the non-linear dirty paper coding (DPC) [7]. However, linear alternatives are more attractive in terms of complexity. For instance, zero-forcing (ZF) linear precoding performs close to DPC in case many users are available and optimal user scheduling is performed [8]. By relaxing the zero interference constraint at each user, it has been proved that the so-called regularized channel inversion (a MMSE-like precoder) can significantly improve the performance [9].

As for the return link, the maximum sum-rate is known to be achieved via successive interference cancelation with minimum mean-squared error filtering at each stage (MMSE-SIC) [10]. However, much simpler, linear alternatives, such as the zero-forcing (ZF) receiver or the plain MMSE receiver $([11,12])$ are also popular because of their lower computational complexity.

Recently, the performance of the return link of a full onground architecture was investigated in [13], featuring an adaptive coding and modulation (ACM) enhanced DVBRCS physical layer. Results showed an increase in throughput at the cost of some loss in availability when linear MMSE was applied; with MMSE-SIC, a significant improvement in throughput and availability was reported. An equivalent analysis for the forward link was reported in [3], where again a throughput increase was achieved at the expense of a loss in availability.

Within the SatNEx III (Satellite Network of Experts) framework, funded by the European Space Agency, and building on previous results such as those above, the use of multiuser interference mitigation schemes together with GBBF was analyzed to evaluate its potential improvement with respect to more classic onboard beamforming settings. The main achievements of this research, which have been partly presented in $[14,15]$, are reported in detail in this article. It will be shown that full on-ground architectures tend to outperform hybrid architectures with fixed on-board weights and on-ground multiuser interference mitigation. This conclusion will be analytically supported under perfect channel state information (CSI), and a sufficient condition for the two architectures to be equivalent will be given. Moreover, detailed simulations will provide insight on the behavior of both architectures when imperfect channel knowledge is assumed, and also when the analog beamforming suffers from miscalibration issues.

The rest of the article is structured as follows: Section 2 describes the system model, Section 3 evaluates the performance of some processing techniques when the gateway has perfect CSI, Section 4 describes the modeling of non-perfect CSI, Section 5 reports simulation results and, finally, conclusions are summarized in Section 6.

Notation: Boldface uppercase letters denote matrices and boldface lowercase letters refer to column vectors. We denote by $(.)^{H}$ the Hermitian transpose. The $N \times N$ identity matrix is denoted by $\mathbf{I}_{N}$, and $\operatorname{diag}(\mathbf{a})$ builds a diagonal matrix from the elements of the vector a. Nonboldface lowercase letters are used to refer to the entries of a matrix: the $(k, l)$ th entry of the matrix $\mathbf{W}$ is denoted by $w_{k l}$.

\section{System description}

The object of study consists of a single satellite which gives service to a region covered by $K$ beam spots; a single user link is active at a given time and carrier block at each beam. The satellite in Figure 1 uses a fed reflector antenna array with $N$ feeds to exchange signals with the users. In the absence of on-board beamforming, all these signals will be relayed through a feeder link with the gateway station (GW) on Earth. In the sequel, we will assume a single gateway and neglect the possible impairments caused by the feeder link. As a more conventional option, if on-board beamforming is applied, beamforming weights will be assumed to be fixed and $K$ signals, one per beam, will be synthesized from the combination of the $N>K$ feed signals, with the corresponding reduction in the feeder link required capacity. For the radiation pattern which will be considered, only a small subset of the feeds will be involved in the conformation of each individual beam. As a limit case with practical application in some cases, for $N=K$ each radiating element feeds a different beam, and the two options considered in this article collapse to the same case.

We will refer to the full on-ground processing as feed processing, whereas the hybrid architecture with on-board fixed beamforming will be often quoted as beam processing.

\subsection{Return link}

Let $K$ be the number of users on Earth and $N$ is the number of on-board feeds. At the feed level, the mathematical model of the return link reads

$$
\mathbf{y}=\mathbf{H s}+\mathbf{n}
$$

where $\mathbf{y}$ is an $N \times 1$ vector that contains the symbols received at each feed, $\mathbf{s}$ is a $K \times 1$ stack of the symbols transmitted by each user (see Figure 2), $\mathbf{n}$ is the $N \times 1$ vector of zero-mean complex white Gaussian noise, such that $\mathbb{E}\left\{\mathbf{n n}^{H}\right\}=N_{o} \cdot \mathbf{I}_{N}$, and $\mathbf{H}$ represents the $N \times$ 


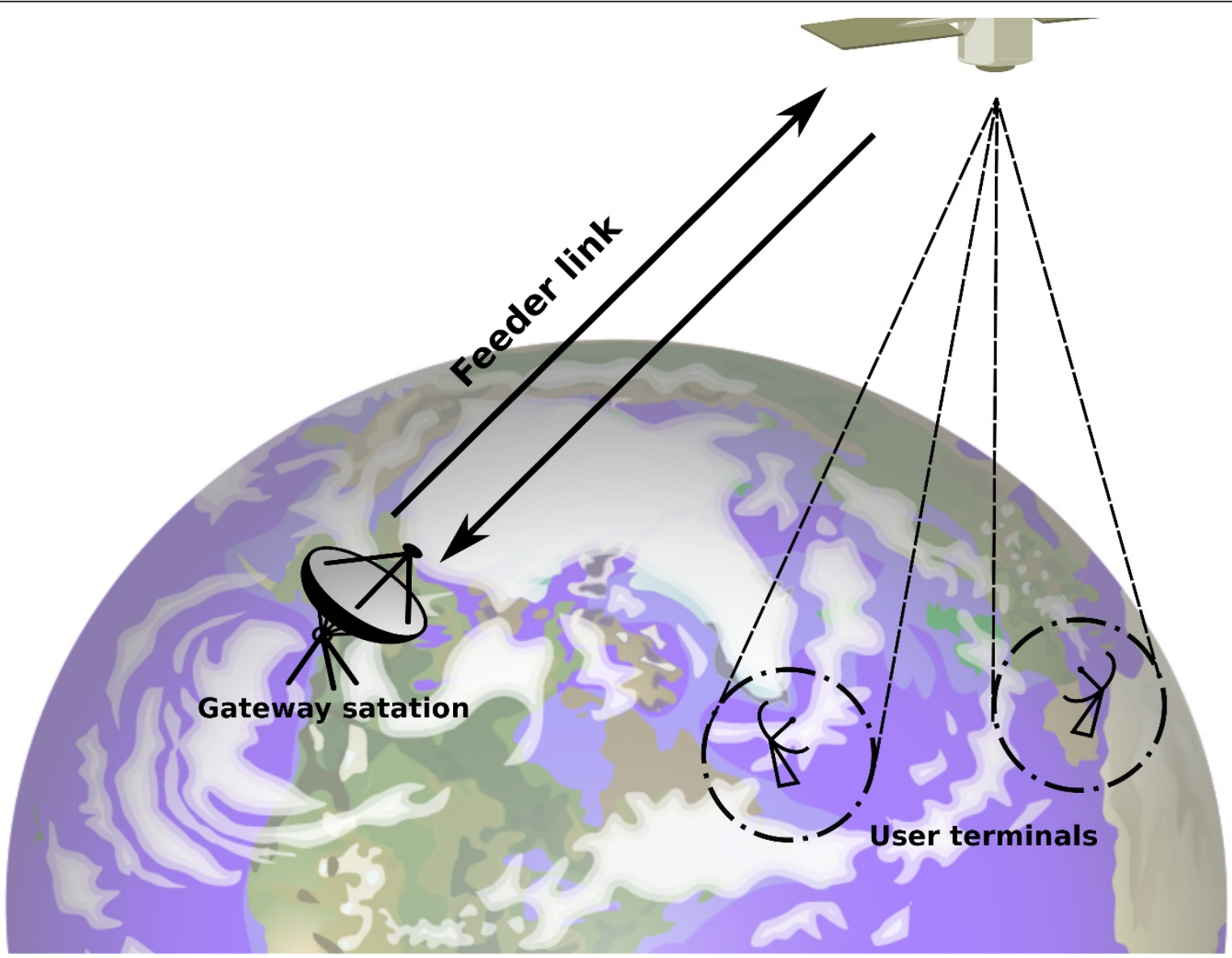

Figure 1 Descriptive diagram of a multibeam satellite system.

$K$ channel matrix. The channel flat-frequency response is parameterized as

$$
\mathbf{H}=\mathbf{G L}
$$

where each matrix is described in the following paragraphs.

Feed radiation pattern and path losses. $\mathbf{G}$ is assumed to be an $N \times K$ matrix that accounts for the gains of the feed radiation pattern, the on-board attenuation and the free space losses; recall that the feeder link is considered transparent. Matrix $\mathbf{G}$ is not deterministic given the random positions of the users within their corresponding beam spots.

Atmospheric fading. The attenuation due to atmospheric phenomena-specially the rain-can be significant in bands such as the Ka-band. In this study, the empirical probability density function (pdf) of this attenuation, obtained for the city of Rome, was used [16]. This pdf would define the statistics of the marginal distribution of each link's attenuation, but it reports no information about the possible spatial correlation.
This spatial correlation is of great importance for the design of multi-satellite systems as the one addressed in [17], and its influence in a general random MIMO channel has been covered in [18] but, as far as the authors know, little has been said about rain correlation in multibeam satellite systems when there is only one user per beam. In [19], the concept of correlated area (CA) was introduced, defined as a spatial region in which Earth stations experiment highly correlated rain attenuation; the correlation with stations out of the CA would be considered negligible. The shape and length of a CA depends on many environmental factors, but diameters between 30 and $50 \mathrm{~km}$ are quoted to be frequent.

Based on these figures, it seems reasonable to assume that each beamspot belongs to a different CA when the radius of the spots is large enough. This was done, for instance, in [20], where beamspots have a diameter of 250 $\mathrm{km}$ and therefore users from different cells are assumed to be uncorrelated in terms of rain fading. In our case, the beam radius is also large (100 beams to cover Europe), and correlation values have been proven to be negligible, 


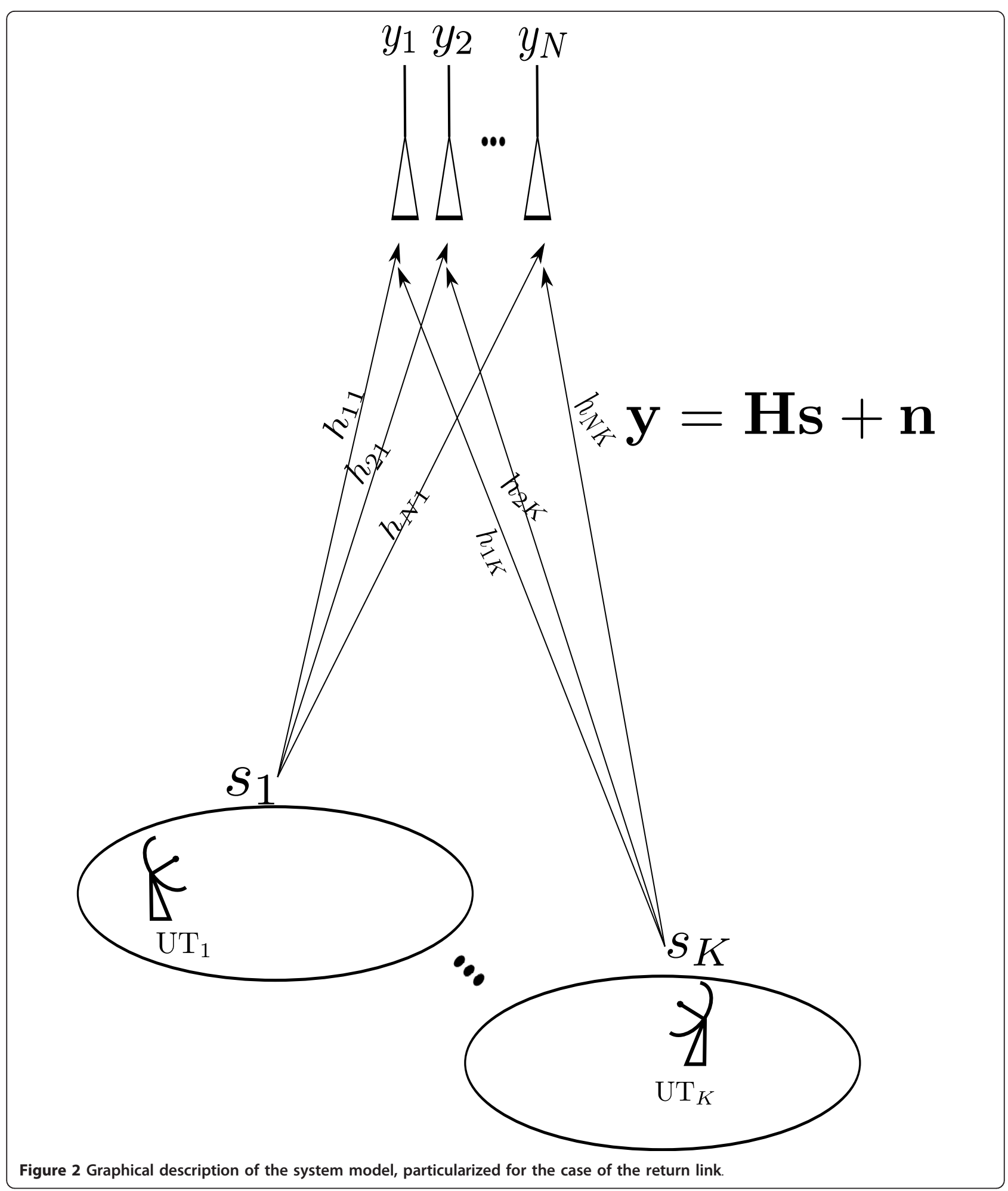

as shown in Appendix 1. As a consequence, matrix $\mathbf{L}$ is assumed to be diagonal and its entries are considered independent.
Further refinements of the channel model were not considered since this simple characterization has been deemed to be useful for the intended comparisons. 
In those cases for which a fixed beamforming is applied on-board, the above model must be modified accordingly. Let $\mathbf{B}$ the $K \times N$ beamforming matrix and define $\mathbf{H}_{b} \doteq \mathbf{B H}$ and $\mathbf{n}_{b} \doteq \mathbf{B n}$, then the received signal becomes

$$
\mathbf{y}_{b}=\mathbf{H}_{b} \mathbf{s}+\mathbf{n}_{b}
$$

Note that $\Sigma \doteq \mathbb{E}\left\{\mathbf{n}_{b} \mathbf{n}_{b}^{H}\right\}=N_{o} \cdot \mathbf{B B}^{H}$.

\subsection{Forward link}

Analogously to the return link, we describe the signal model for the forward link, which reads as

$$
\mathbf{r}=\mathbf{H}^{T} \mathbf{x}+\mathbf{w}
$$

where $\mathbf{r}$ (resp. w) is a $K \times 1$ vector containing the stack of the received signals (resp. noise components) at each user. Similarly as before, we assume that $\mathbb{E}\left\{\mathbf{w w}^{H}\right\}=N_{o} \cdot \mathbf{I}_{K}$. The $N \times 1$ vector $\mathbf{x}$ is the stack of the transmitted signals at all feeds. The forward link channel matrix is simply the transpose of that of the return link: $\mathbf{H}^{T}$ is of size $K \times N$. Let us stress that this reciprocity is strictly limited to the mathematical formalism of both links. In fact, the reciprocity between the forward and return links does not hold in practice, since they typically involve different frequency bands.

For a fair comparison of all forward link scenarios that will be considered in the sequel, it is critical to define a common transmit power constraint. For this, we assume the following constraint on the average power transmitted at the feed level:

$$
\mathbb{E}\left\{\mathbf{x}^{H} \mathbf{x}\right\} \leq P_{T}
$$

where $P_{T}$ denotes the total transmit power.

In the case of a fixed on-board beamforming, we have that $\mathbf{x}=\mathbf{B}^{T} \mathbf{x}_{b}$, where $\mathbf{x}_{b}$ is the stack of the on-ground transmitted signal in the beam space, keeping in mind that a perfectly calibrated and noiseless feeder link is assumed. The signal model (4) becomes

$$
\mathbf{r}=\mathbf{H}_{b}^{T} \mathbf{x}_{b}+\mathbf{w}
$$

where $\mathbf{H}_{b} \doteq \mathbf{B H}$ was defined above and expresses the principle of beamforming: the effect of the matrix $\mathbf{B}$ is essentially the linear combination of the radiation pattern of all $N$ feeds to generate $K$ beam radiation patterns.

\section{Perfect CSI at the gateway}

As a first step, let us assume that the gateway has perfect knowledge of the channel state, when acting either as transmitter or receiver. Throughout this section, we will establish measures of performance and show that, under linear combining, feed processing outperforms beam processing.

\subsection{Return link}

For the return link, and assuming on-ground feed processing, the MMSE combiner [21] that yields $\hat{\mathbf{s}}=\mathbf{W}^{H} \mathbf{y}$ is

$$
\mathbf{W}^{H}=\mathbf{H}^{H}\left(N_{0} \mathbf{I}_{N}+\mathbf{H H}^{H}\right)^{-1}=\left(N_{0} \mathbf{I}_{K}+\mathbf{H}^{H} \mathbf{H}\right)^{-1} \mathbf{H}^{H}
$$

whereas the processing of the beams would entail $\hat{\mathbf{s}}=\mathbf{W}_{b}^{H} \mathbf{y}_{b}$ with

$$
\mathbf{W}_{b}^{H}=\left(\mathbf{I}_{K}+\mathbf{H}_{b}^{H} \Sigma^{-1} \mathbf{H}_{b}\right)^{-1} \mathbf{H}_{b}^{H} \Sigma^{-1} .
$$

A key objective of this study is to compare the performance of these two approaches. To accomplish this task, we will make use of the mean-squared error (MSE) after combining, which is defined as $\mathbb{E}\left\{|\mathbf{s}-\hat{\mathbf{s}}|^{2}\right\}$.

Let us denote $\mathbf{Q}_{f}$ its covariance matrix, then it would read

$$
\mathbf{Q}_{f}=\mathbf{R}_{x}-\mathbf{R}_{x y} \mathbf{R}_{y}^{-1} \mathbf{R}_{y x}=\left(\mathbf{I}_{K}+\frac{1}{N_{0}} \mathbf{H}^{H} \mathbf{H}\right)^{-1}
$$

for the case of feed processing. For the case of beam processing, it would be

$$
\mathbf{Q}_{b}=\left(\mathbf{I}_{k}+\mathbf{H}_{b}^{H} \frac{\left(\mathbf{B B}^{H}\right)^{-1}}{N_{0}} \mathbf{H}_{b}\right)^{-1}=\left(\mathbf{I}_{K}+\frac{1}{N_{0}} \mathbf{H}^{H} \mathbf{P H}\right)^{-1}
$$

with $\mathbf{P}=\mathbf{B}^{H}\left(\mathbf{B B}^{H}\right)^{-1} \mathbf{B}$.

Since the SINR for the $i$ th user is given by $1 / \mathbf{Q}_{i i}-1$ [11], it makes sense to use the total MSE, given by $\sum_{i=1}^{K} \mathbf{Q}_{i i}=\operatorname{trace}\{\mathbf{Q}\}$, as a performance metric. Then, it can be shown that

$$
\operatorname{trace}\left\{\mathbf{Q}_{b}\right\} \geq \operatorname{trace}\left\{\mathbf{Q}_{f}\right\}
$$

as follows. Let us express both traces as

$$
\operatorname{trace}\left\{\mathbf{Q}_{f}\right\}=\sum_{i=1}^{K} \lambda_{i}\left(\mathbf{Q}_{f}\right)=\sum_{i=1}^{K} \frac{N_{0}}{N_{0}+\lambda_{i}\left(\mathbf{H}^{H} \mathbf{H}\right)}
$$

and

$$
\operatorname{trace}\left\{\mathbf{Q}_{b}\right\}=\sum_{i=1}^{K} \lambda_{i}\left(\mathbf{Q}_{b}\right)=\sum_{i=1}^{K} \frac{N_{0}}{N_{0}+\lambda_{i}\left(\mathbf{H}^{H} \mathbf{P H}\right)}
$$

where $\lambda_{i}\left(\mathbf{H}^{H} \mathbf{H}\right)$ denotes the $i$ th largest eigenvalue of $\mathbf{H}^{H} \mathbf{H}$. We have that (11) is an immediate consequence of the following, stronger result.

Theorem 1 Let $\mathbf{H}$ and $\mathbf{B}^{H}$ be two tall matrices of the same size with full column rank. Let $\mathbf{P}$ be a projection matrix of $\mathbf{B}$, that is, $\mathbf{P}=\mathbf{B}^{H}\left(\mathbf{B B}^{H}\right)^{-1} \mathbf{B}$. Then, it holds that 
$\sigma_{i}(\mathbf{H}) \geq \sigma_{i}(\mathbf{P H})$, with $\sigma_{i}(\mathbf{A})$ denoting the ith largest singular value of matrix $\mathbf{A}$.

For the proof, see Appendix 2.

A sufficient condition for the traces to be equal is that $\mathbf{P H}=\mathbf{H}$. Since $\mathbf{P}$ is a projection matrix, this will happen whenever range $\left(\mathbf{B}^{H}\right)=\operatorname{range}(\mathbf{H})$. However, since $\mathbf{B}$ is fixed and $\mathbf{H}$ time-varying, it seems not possible to meet such condition. Recall that, even if the fading is negligible, the users are assumed to be located randomly into their beam spots. Thus, assuming constant $\mathbf{H}$ would require the feed pattern to be constant over each cell; it would also require users near the border of their cells to experiment almost the same interference as if they were located close to the center. On account of all these facts, ensuring $\operatorname{range}\left(\mathbf{B}^{H}\right)=\operatorname{range}(\mathbf{H})$ is not realistic.

\subsection{Forward link}

As mentioned above, interference mitigation techniques in the forward link take the form of precoding at the gateway. In this article, we focus exclusively on linear precoding. In the case of on-ground feed processing, linear precoding is expressed as

$$
\mathbf{x}=\mathbf{F s}
$$

with $\mathbf{F}$ the $N \times K$ precoding matrix, and $\mathbf{s}$ the $K \times 1$ symbol vector. The $k$ th entry of $\mathbf{s}$ is the unit energy constellation symbol destined to the $k$ th user. To comply with the transmit power constraint (5), the precoding matrix $\mathbf{F}$ has to satisfy

$$
\text { trace }\left\{\mathbf{F F}^{H}\right\} \leq P_{T} \text {. }
$$

In the case of adaptive linear precoding in the beam space, we write

$$
\mathbf{x}_{b}=\mathbf{F}_{b} \mathbf{S}
$$

where $\mathbf{F}_{b}$ is the $K \times K$ precoding matrix, in terms of which the transmit power constraint (5) becomes

$$
\text { trace }\left\{\mathbf{B}^{T} \mathbf{F}_{b} \mathbf{F}_{b}^{H} \mathbf{B}^{*}\right\} \leq P_{T} .
$$

In this section, we assume a zero-forcing (ZF) precoder. The zero-forcing (ZF) criterion targets the complete cancelation of the inter-user interference, by precoding with the pseudoinverse of the channel matrix. The corresponding expressions are

$$
\begin{aligned}
& \mathbf{F}=\sqrt{\beta} \mathbf{H}^{*}\left(\mathbf{H}^{T} \mathbf{H}^{*}\right)^{-1} \\
& \mathbf{F}_{b}=\sqrt{\beta_{b}} \mathbf{H}_{b}^{*}\left(\mathbf{H}_{b}^{T} \mathbf{H}_{b}^{*}\right)^{-1}
\end{aligned}
$$

for the precoding in the feed space and beam space, respectively. The value of the constants $\beta$ and $\beta_{b}$ has to be chosen such to comply with (15) and (17), respectively. Note that these particular versions of the ZF linear precoders are such that they equalize the signal to noise ratio (SNR) among users. The resulting SNR is given by

$$
\begin{aligned}
& \mathrm{SNR}_{f}=\frac{P_{T} / N_{0}}{\operatorname{trace}\left\{\left(\mathbf{H}^{T} \mathbf{H}^{*}\right)^{-1}\right\}}=\frac{P_{T} / N_{0}}{\operatorname{trace}\left\{\left(\mathbf{H}^{H} \mathbf{H}\right)^{-1}\right\}} \\
& \mathrm{SNR}_{b}=\frac{P_{T} / N_{0}}{\operatorname{trace}\left\{\mathbf{B}^{T}\left(\mathbf{B}^{*} \mathbf{H}^{*} \mathbf{H}^{T} \mathbf{B}^{T}\right)^{-1} \mathbf{B}^{*}\right\}}=\frac{P_{T} / N_{0}}{\operatorname{trace}\left\{\mathbf{B}^{H}\left(\mathbf{B} \mathbf{H H}^{H} \mathbf{B}^{H}\right)^{-1} \mathbf{B}\right\}}
\end{aligned}
$$

for the feed and beam processing, respectively. We prove here that the SNR achieved by the feed processing is always greater than or equal to that associated with the beam processing: $\mathrm{SNR}_{f} \geq \mathrm{SNR}_{b}$. This is a direct consequence of the following property.

Theorem 2 Let $\mathbf{H}$ and $\mathbf{B}^{H}$ be two tall matrices of the same size with full column rank. Then, the following inequality holds:

$$
\operatorname{trace}\left\{\left(\mathbf{H}^{H} \mathbf{H}\right)^{-1}\right\} \leq \operatorname{trace}\left\{\mathbf{B}^{H}\left(\mathbf{B} \mathbf{H} \mathbf{H}^{H} \mathbf{B}^{H}\right)^{-1} \mathbf{B}\right\} \text {. }
$$

The equality is reached if $\mathbf{H}$ and $\mathbf{B}$ share the same left and right singular vectors, respectively.

For the proof, see Appendix 3.

As stated in the theorem, a sufficient condition for the beam processing not to suffer any performance loss with respect to the feed processing is that the matrices $\mathbf{H}$ and $\mathbf{B}$ share the same left and right singular vectors, respectively. However, in a similar way as for the return link, this condition is not likely to be met in practice since $\mathbf{B}$ is a fixed (non channel-adaptive) beamforming matrix while $\mathbf{H}$ is time-varying (due to the random characteristic of the users positions).

\section{Non-perfect CSI at the gateway}

In a realistic scenario, the gateway does not know the actual values in the channel matrix $\mathbf{H}$, but has only an estimate of them. The type and quality of these estimates, commonly based on the use of training sequences, will have an effect on the ultimate performance of the system. In this section, the estimation of the matrix $\mathbf{H}$ will be introduced. Some degree of uncertainty on the beamforming matrix $\mathbf{B}$ will also be discussed, while the noise power $N_{0}$ will be assumed perfectly known.

\subsection{Channel estimation by training sequences}

For the estimation of the channel in the return link, each user employs a distinct training sequence, known as its unique word (UW). The gateway, upon reception 
of all the sequences, estimates the values in the channel matrix.

If perfect symbol synchronism can be assumed, then Walsh-Hadamard sequences can be used as UWs, and it is possible to apply the pseudoinverse procedure [13] to estimate the channel matrix. However, due to the nature of the communication in the return link, symbol synchronism cannot be assumed.

In spite of this fact, the pseudoinverse procedure can still be used as long as good timing and frequency recovery is applied. Channel estimation under these circumstances would require the use of pseudorandom sequences with good cross-correlation properties, rather than Walsh-Hadamard sequences. Moreover, under accurate synchronization, pseudorandom sequences have been reported [13] to produce negligible correlation between the estimation errors of the different elements of the matrix.

On account of the previous statements, and in the absence of a fixed beamforming, channel estimation would be modeled as

$$
\hat{\mathbf{H}}=\mathbf{H}+\mathbf{E}=\mathbf{H}+\frac{N_{0}}{L} \mathbf{W}
$$

where $L$ is the training sequence length and $\mathbf{W}$ is a matrix with independent zero-mean unit-variance Gaussian entries. For the case with beamforming on-board, it would read

$$
\hat{\mathbf{H}}_{b}=\mathbf{H}_{b}+\mathbf{E}_{b}=\mathbf{H}+\frac{N_{0}}{L} \mathbf{B W} .
$$

In the forward link, the symbol synchronicity is not an issue anymore. We consider that the precoder design is now based on a feed channel estimate $\hat{\mathbf{H}}^{T}=\mathbf{H}^{T}+\mathbf{E}$ (or $\hat{\mathbf{H}}_{b}^{T}=\mathbf{H}_{b}^{T}+\mathbf{E}_{b}$ in the beam space). Each row of $\hat{\mathbf{H}}^{T}$ (or $\hat{\mathbf{H}}_{b}^{T}$ ) is based on a channel estimation which is carried out separately at each user terminal and then reported to the gateway via a return channel (assumed ideal). Note that the reporting of $\hat{\mathbf{H}}^{T}$ to the gateway implies feeding back $(N-K) K$ more channel samples than for reporting $\hat{\mathbf{H}}_{b}^{T}$. We assume $L$-length orthogonal training sequences, such that the entries of $\mathbf{E}$ (or $\mathbf{E}_{b}$ ) are i.i.d. zero mean complex circular symmetric Gaussian random variables with variance inversely proportional to $L$.

\subsection{Analog miscalibration}

For the case of beam processing in the return link, the gateway must be aware of the exact beamforming weights that are set on board, since they will be necessary to compute the noise covariance matrix in the MMSE combiner (8). Even though these are subject of calibration, it is very likely that their actual values will experiment some minor changes through time, mainly because of the non-ideal nature of the analog circuitry. As a consequence, the information at the gateway can be seen as an estimate of the actual beamformer. The following mathematical model is proposed:

$$
\hat{\mathbf{B}}=\mathbf{B}+\Delta \mathbf{B}
$$

where the entries in $\Delta \mathbf{B}$ are of the form $\Delta \mathbf{B}_{i j}=b_{i j} r_{i j}$ and $r_{i j}$ are independent, real, zero-mean Gaussian random variables. This models a variation, both in the real and imaginary parts of the weights, that is random with given variance, but proportional to the original value.

\section{Simulation results}

In order to further compare the performance of the proposed precoding and multiuser detection architectures, Monte Carlo simulations have been carried out according to the scenario described in Table 1. This scenario features $K=100$ beams covering the whole Europe area. The satellite antenna pattern was provided by ESA, and corresponds to an array fed reflector antenna with $N=$ 155 feeds. Matrix B was also provided by ESA, as a typical beamforming matrix of current systems. It was designed such as to limit the level of interference among users in a conventional system (without interference mitigation technique but with adequate frequency reuse pattern).

The user link has a total available bandwidth of 500 $\mathrm{MHz}$; color schemes with frequency reuse factor equal to 3 and 1 were studied, corresponding to 166 and 500 $\mathrm{MHz}$ available bandwidth per beam, respectively. The reference scenario consists in a frequency reuse factor equal to 3, fixed beamforming and no processing at the gateway. Simulation results have been extracted for a number of interference mitigation techniques, both for the forward link and the return link. The purpose of this is to compare the performance of both architectures in as many different situations as possible. For

\section{Table 1 Simulation parameters}

\begin{tabular}{cc}
\hline \multicolumn{2}{c}{ Simulation parameters } \\
\hline Atmospheric fading & City of Rome [16] \\
UTs location distribution & Uniformly distributed \\
Feed and beam gain patterns & Generated from data provided by ESA \\
FL simulation parameters \\
UT antenna gain $G_{R}^{2}$ & $41.7 \mathrm{dBi}$ \\
UT clear sky $G_{R}^{2} / T$ ClearSky & $17.68 \mathrm{~dB} / \mathrm{K}$ \\
UT rain delta temperature & $221.83 \mathrm{~K}$ \\
RL simulation parameters \\
Training sequence length & $128 \mathrm{symbols}$ \\
Receiver noise figure & $2.5 \mathrm{~dB}$ \\
Total receiver noise temperature & $517 \mathrm{~K}$
\end{tabular}


illustrations purposes simulation results cover a large range of transmit powers, although it is important to stress that the most extreme values do not correspond to practical cases.

Results have been averaged for a total of 1,000 channel realizations, with the exception of those showing the average probability of non-availability, which required 10,000 iterations to yield a reasonable confidence interval. Apart from the fading, the randomness of the channel is due to the position of the users, which are assumed to be uniformly distributed within each spot. For each realization, the SINR for each user after interference mitigation is computed, and its throughput is then inferred according to the preliminary specifications or DVB-RCS2 in the RL and DVB-S2 in the FL.

\subsection{Return link}

The user link operates at $30 \mathrm{GHz}$ (Ka-band), and is based on the DVB-RCS2 standard [22]. The baudrate is $4 \mathrm{Msymb} / \mathrm{s}$ and the guardbands amount to the $11 \%$ of the carrier bandwidth [23]. Apart from the MMSE receiver presented in Section 3.1, the MMSE-SIC receiver has also been simulated, since it is known to be capacity achieving under ideal conditions, and therefore provides an upper bound on the achievable performance.

Figures 3 and 4 depict the evolution of the total average throughput as a function of the terminals EIRP. Results have been averaged only for those realizations in which the link was active. To this extent, Figure 5 shows the average probability of non-availability for the different MUD techniques. It can be observed that a considerable increase in throughput is experimented thanks to using multiuser detection, although at the cost of some loss in availability. In fact, only SIC detection manages to reduce the outage probability with respect to the benchmark scenario.

Moreover, full on-ground processing reports higher throughput figures both with perfect and non-perfect CSI. To further investigate the potential advantage of this strategy, Figure 6 represents the performance gain obtained in this case with respect to the hybrid

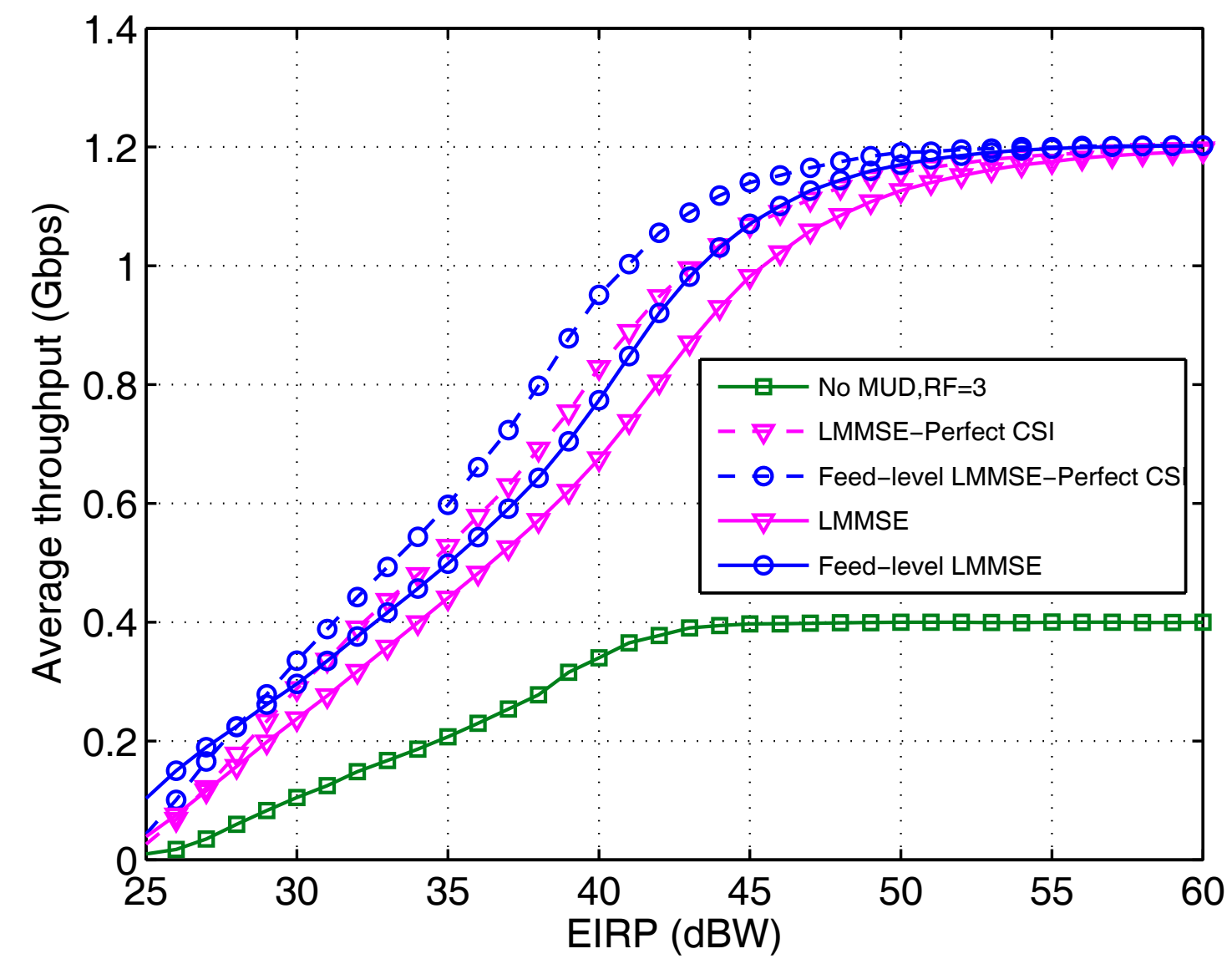

Figure 3 Evolution of throughput as a function of terminal EIRP in the return link, beam processing. 


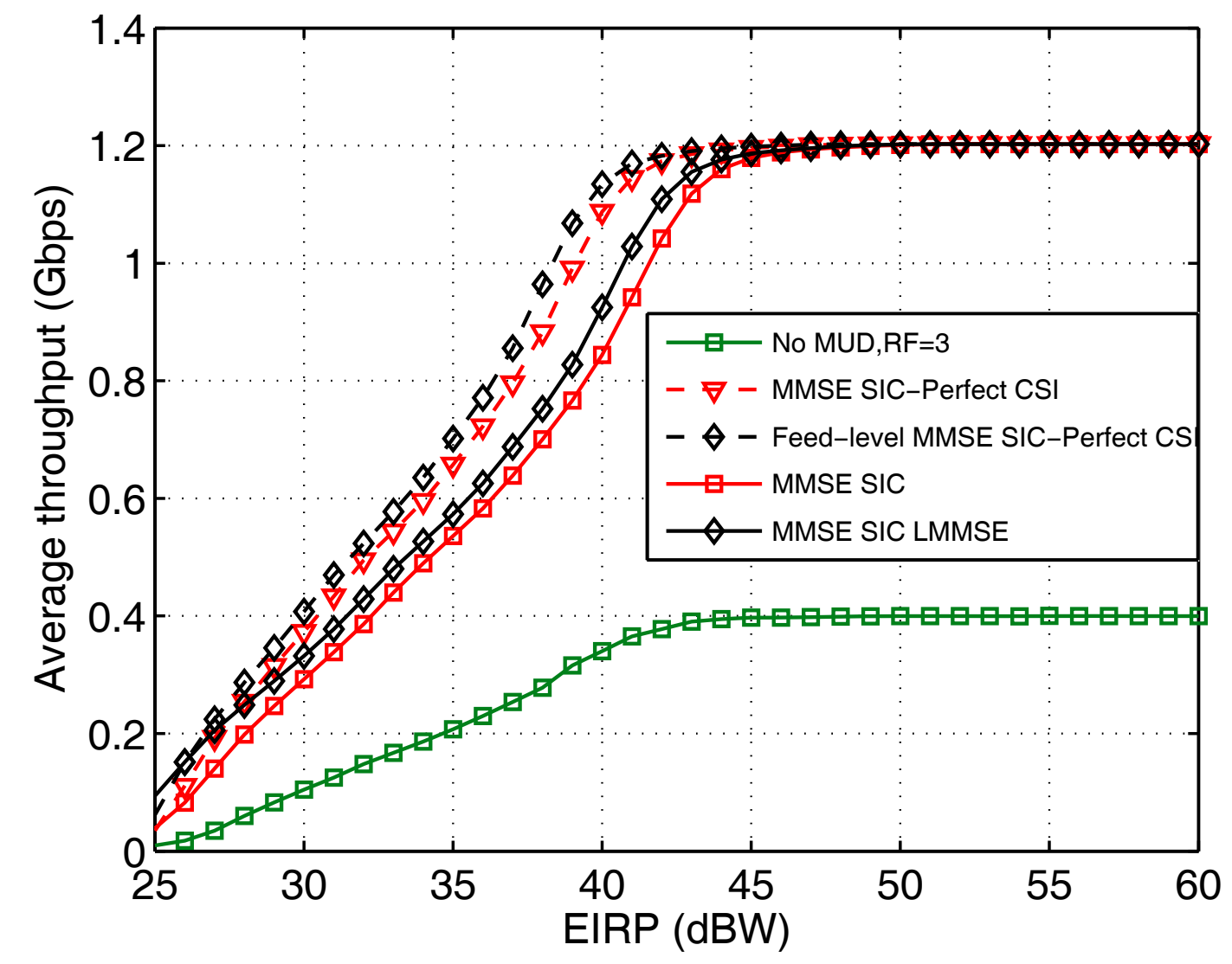

Figure 4 Evolution of throughput as a function of terminal EIRP in the return link, feed processing.

architecture, using training sequences of length 128 symbols. Let $t_{f}$ and $\rho_{f}$ be the average throughput and average availability, respectively, of the overall system when full on-ground feed processing is employed, and define $t b$ and $\rho b$ as the corresponding counterparts in the beam processing case. The feed combining gain is defined as

$$
\gamma \doteq \frac{t_{f} \rho_{f}}{t_{b} \rho_{b}}
$$

It can be seen that, despite the existence of channel estimation errors, there are always non-negligible improvements when choosing a full on-ground architecture. Moreover, results on the hybrid architecture assumed so far perfect knowledge of the fixed beamforming matrix. Recall now the error model (25) for the analog calibration, given by the error matrix $\Delta \mathbf{B}=\left\{b_{i j} r_{i j}\right\}$ and let $\beta$ be the variance of the random variables $r_{i j}$. Following this model, Figure 7 depicts the evolution of the feed combining gain for different values of $\beta$, that is, for different degrees of uncertainty on the analog beamforming weights. Results account for more significant feed combining gains when some degree of uncertainty is present.

\subsection{Forward link}

The user link in the downstream is assumed to operate at $20 \mathrm{GHz}$ (K-band), and is based on the DVB-S2 standard. Besides the ZF precoder presented in Section 3.2, the following advanced precoders are considered and will be compared in the simulations:

- The regularized channel inversion precoding [9].

- The so-called UpConst MMSE precoder which is based on the uplink-downlink duality [24]. This precoder solution was proposed in [3], where it is said to achieve a good compromise between throughput and availability.

Again, simulation results will use the average total throughput and availability as performance measures, but this time the total transmit power $P_{T}$ will be used as a parameter rather than the EIRP since the directivity of the feeds is part of the feed radiation pattern data provided by ESA. 


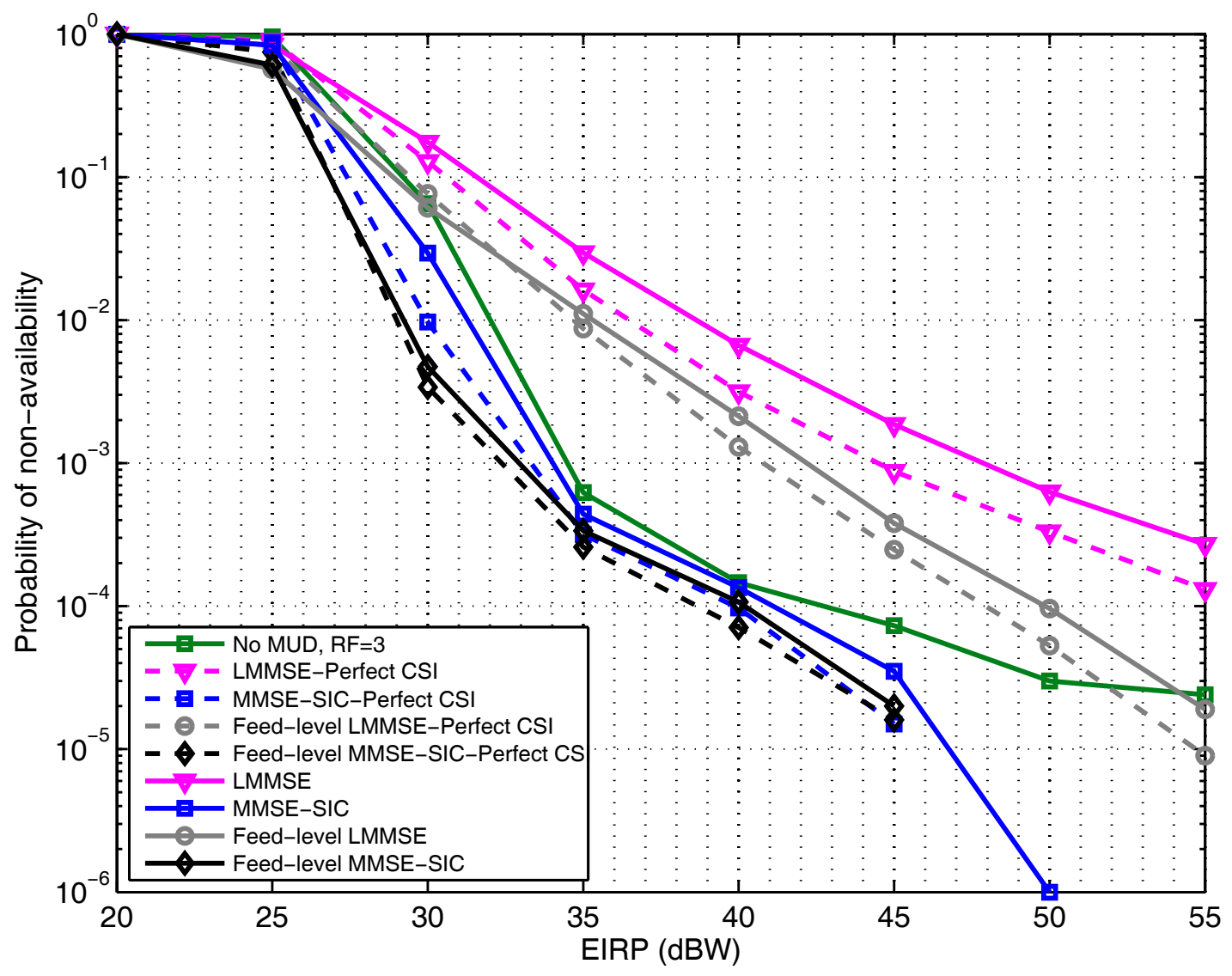

Figure 5 Average probability of non-availability in the return link as a function of terminal EIRP

Let us assume first that the channel is perfectly known at the gateway. Figures 8 and 9 first compare the regularized channel inversion and ZF linear precoder. The regularized channel inversion significantly outperforms the more naive ZF precoder. Most importantly, the benefit of the full on-ground architecture (i.e., feed processing) is apparent both in terms of throughput and availability. For instance, at $P_{T}=30 \mathrm{dBW}$ the regularized channel inversion in the feed space generates a $111 \%$ relative throughput increase with respect to the reference scenario, and $17 \%$ with respect to the same processing in the beam space. However, a slight decrease in system availability can still be observed with respect to the reference scenario. Figures 10 and 11 consider additionally the UpConst MMSE precoder, and illustrate again the benefit of the full on-ground architecture. It can be seen that the comparison between the regularized channel inversion and UpConst MMSE precoders depends on the value of the transmit power: in terms of availability, the regularized channel inversion outperforms the UpConst MMSE precoder for high values of the transmit power, and viceversa at low values of the transmit power.

We now disregard the assumption of perfect CSI at the gateway, and analyze the robustness of the different schemes to channel estimation errors. Figures 12 and 13 depict the achievable throughput and system availability for a training sequence length $L=256$. The full onground architecture still appears beneficial, especially throughput wise. Moreover, from comparing Figures 11 and 13, it can be noticed that the regularized channel inversion appears to be more robust to imperfect CSI than the precoder based on the uplink-downlink duality.

In Figure 14, we compare the feed combining gain (26) associated with the regularized channel inversion for different degree of CSI: perfect CSI, imperfect CSI with $L=1024$, and $L=256$. We can observe the robustness of the feed processing with regularized channel inversion. In fact, quite surprisingly, for moderate to high values of the transmit power the relative gain 


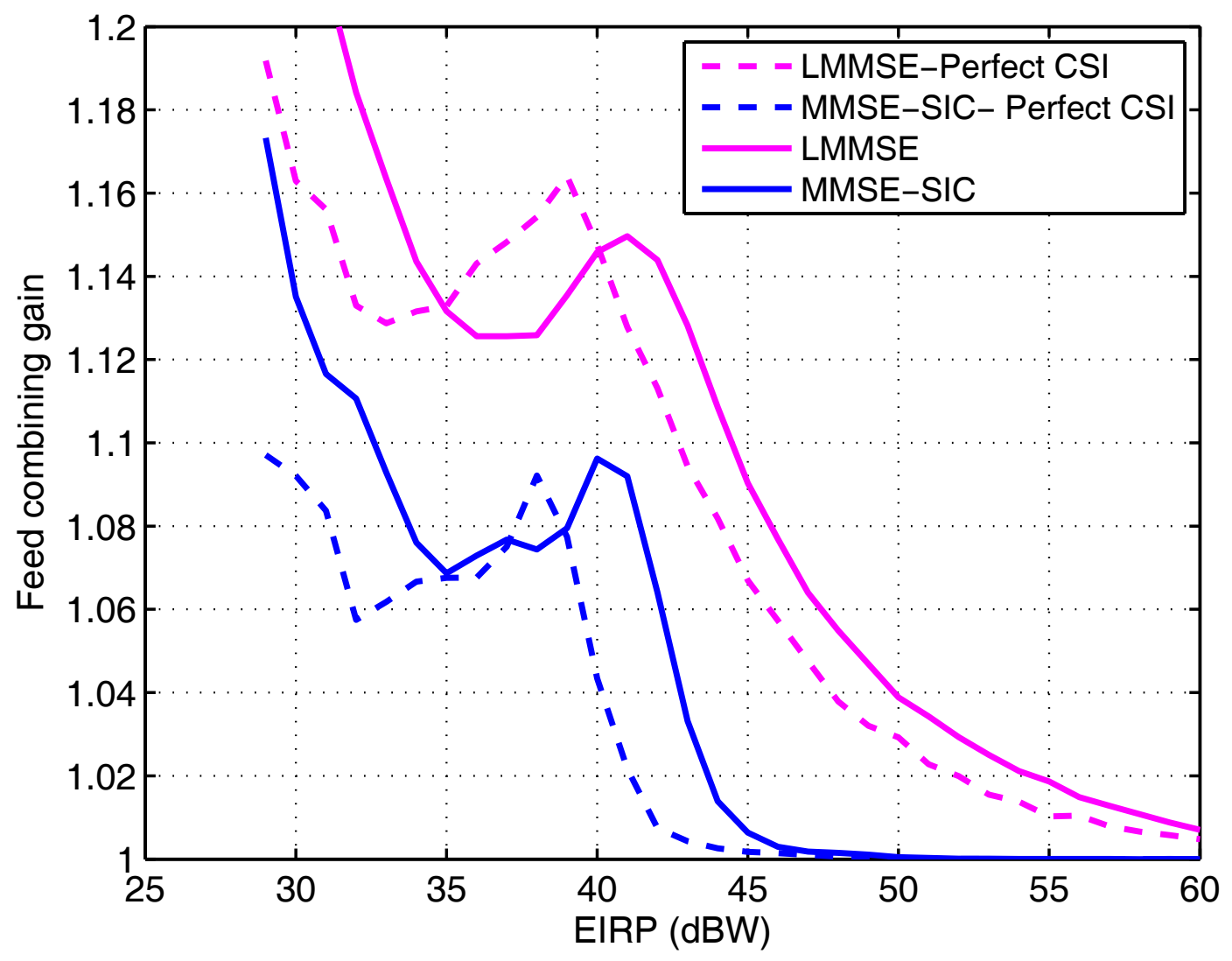

Figure 6 Feed combining gain $\gamma$ for the return link as a function of terminal EIRP

generated by the full on-ground architecture for this precoder increases as the degree of CSI decreases, reaching $24 \%$ with $P_{T}=30 \mathrm{dBW}$ and $L=256$ symbols.

\section{Conclusions}

The results obtained in the previous sections show that feed-level techniques tend to outperform beam processing ones, a fact that has been analytically proved for the case of perfect CSI. In the particular case of the return link, this gain may be rather small when the channel estimation errors are noticeable but the estimation of the on-board analog beamforming is accurate. On the contrary, if the uncertainty on the beamforming is high, then the advantage for using all the information from the feeds seems to be much higher: with the greatest level of uncertainty simulated, the feed combining gain reaches $20 \%$ at an EIRP $40 \mathrm{dBW}$. In what refers to the forward link, the uncertainty about the analog beamforming is not relevant, and the feed processing gain amounts to $24 \%$ with $P_{T}=30 \mathrm{dBW}$ and a realistic level of CSI. It is to be noticed, however, that working with the 155 feed signals would require rather more bandwidth in the feeder link. Therefore, there exists a tradeoff between performance and feeder link requirements, and the choice of the most suitable processing architecture would need to take into account all these considerations.

\section{Appendix 1: Spatial correlation of rain fading}

Next, we present a simple model for the rain fading correlation between two links to assess its potential impact on the interference mitigation schemes. To start with, we will follow the well-known model which specifies that the joint distribution of rain attenuation on two slant paths, which we will call $A_{1}$ and $A_{2}$, is lognormal and presents the following correlation factor [25]

$$
r=\frac{e^{c(d) \sigma_{1} \sigma_{2}}-1}{\sqrt{\left(e^{\sigma_{1}^{2}}-1\right)\left(e^{\sigma_{2}^{2}}-1\right)}}
$$

where $d$ is the horizontal distance between both points on Earth, $\sigma_{i}^{2}$ is the variance of the marginal distribution of $A_{i}$ and $c(d)$ is the correlation factor between 


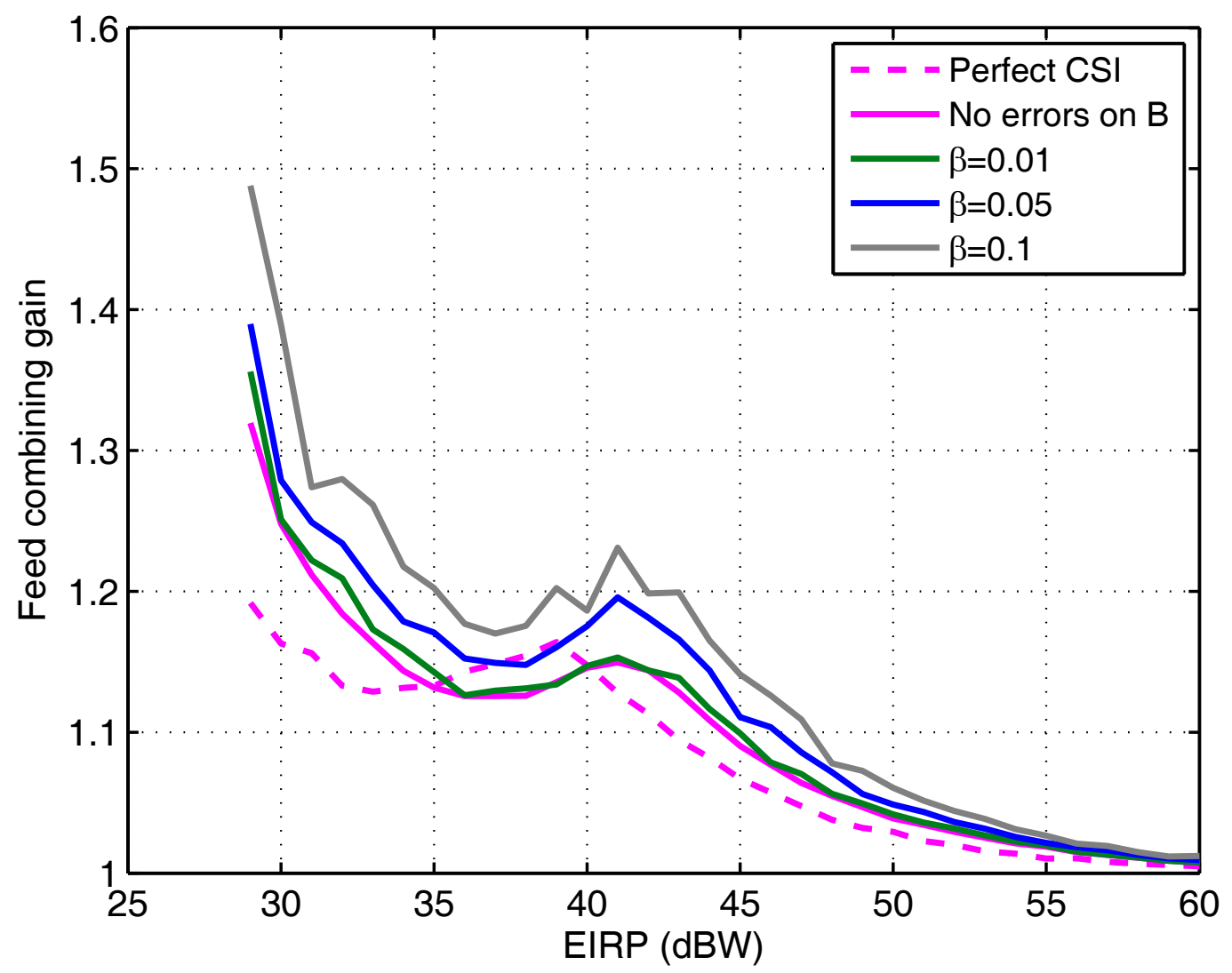

Figure 7 Feed combining gain $\gamma$ for the return link, with imperfect beamforming knowledge, as a function of terminal EIRP

the rain rates. According to [26], this factor may be accurately modeled as

$$
c(d)=e^{-\left(\frac{d}{d_{0}}\right) s_{0}}
$$

where $d_{0}$ is the distance at which $c(d)=1 / e$ (usually called decorrelation distance) and $s_{0}$ is a shape parameter. Although values for both variables must be set according to detailed environmental data, in [26] it has been shown that, for the instantaneous correlation between two points, $s_{0} \approx 1$, yielding

$$
c(d)=e^{-\frac{d}{d_{0}}} .
$$

As for $d_{0}$, different values have been proposed in the literature and, in any case, we must note that these will be highly dependent on the geographical area. For instance, in articles devoted to terrestrial communications like $[27,28]$, values around $d_{0}=0.46 \mathrm{~km}$ are reported, while [25] uses $d_{0}=1.844 \mathrm{~km}$; [26] even reports values up to $d_{0}=7 \mathrm{~km}$ for the State of Oklahoma, although this value was obtained by averaging over a very long period of time.

We will now perform some simulations in order to check the evolution of $r$ with respect to $d$ for different values of $d_{0}$. Since, as stated above, we have assumed the same statistics for the marginal attenuations over each path, Equation (27) simplifies to

$$
r=\frac{e^{c(d) \sigma^{2}}-1}{e^{\sigma^{2}}-1} .
$$

The results obtained are shown in Figure 15, where $\sigma^{2}$ $=1.58$. As we can see, the correlation factor quickly runs close to zero as the distance increases. In Figure 16 , the same data is represented in log scale, in order to interpret such small values; the range of distances has also been multiplied by four. We can see that, even for the (far) most pessimistic case, the correlation values are very small, if not close to zero, after $d=60 \mathrm{~km}$. 


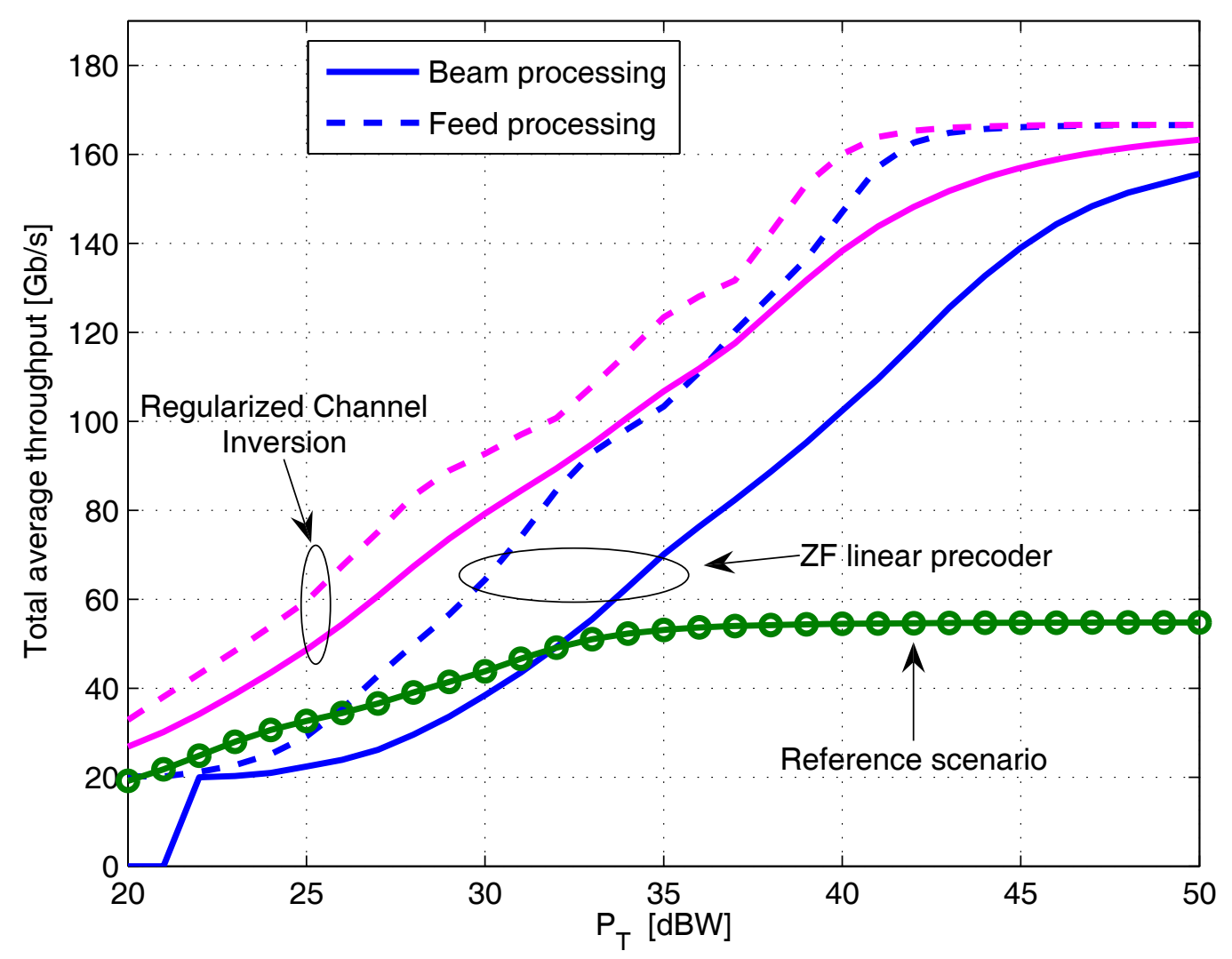

Figure 8 Regularized channel inversion and ZF linear precoding in beam and feed space: throughput comparison with perfect CSI as a function of $P_{T}$.

\section{Appendix 2: Proof of Theorem 1}

The goal is to prove

$$
\sigma_{i}(\mathbf{H}) \geq \sigma_{i}(\mathbf{P H})
$$

with $\sigma_{i}(\mathbf{A})$ representing the $i$ th largest singular value of matrix $\mathbf{A}$.

Consider now $\mathbf{B}=\mathbf{U} \Sigma \mathbf{V}^{H}$, the singular value decomposition (SVD) of $\mathbf{B}$, then

$$
\mathbf{P}=\mathbf{B}^{H}\left(\mathbf{B B}^{H}\right)^{-1} \mathbf{B}=\mathbf{V} \Sigma^{H}\left(\Sigma \Sigma^{H}\right)^{-1} \Sigma \mathbf{V}^{H}=\mathbf{V}\left(\begin{array}{rr}
\mathbf{I}_{K} & 0 \\
\mathbf{0} & \mathbf{0}
\end{array}\right) \mathbf{V}^{H}=\mathbf{V} \boldsymbol{\Phi} \mathbf{V}^{H} .
$$

Since $\mathbf{V}$ is a unitary matrix, then it holds that

$$
\sigma_{i}(\mathbf{V H})=\sigma_{i}(\mathbf{H})
$$

and

$$
\sigma_{i}\left(\mathbf{V}^{H} \boldsymbol{\Phi} \mathbf{V H}\right)=\sigma_{i}(\boldsymbol{\Phi} \mathbf{V H})
$$

Let us define now $\mathbf{A} \doteq \mathbf{V H}$. On account of the previous statements, proving (31) is equivalent to proving

$$
\sigma_{i}(\mathbf{A}) \geq \sigma_{i}(\boldsymbol{\Phi} \mathbf{A})
$$

that is, each singular value of a matrix $\mathbf{A}$ is larger or equal to that of the same matrix after setting some rows to zero. To prove this fact, we will make use of the following property: let $\mathbf{A}$ be in general any tall matrix such that $\mathbf{A}=\left[\begin{array}{llll}\mathbf{a}_{1} & \mathbf{a}_{2} & \ldots & \mathbf{a}_{k}\end{array}\right]$ and define $\mathbf{A}_{r}=\left[\begin{array}{llll}\mathbf{a}_{1} & \mathbf{a}_{2} & \ldots & \mathbf{a}_{r}\end{array}\right]$, then for all $r$ from 1 to $k-1$ it holds that [29]

$$
\sigma_{1}\left(\mathbf{A}_{r+1}\right) \geq \sigma_{1}\left(\mathbf{A}_{r}\right) \geq \sigma_{2}\left(\mathbf{A}_{r+1}\right) \geq \ldots \geq \sigma_{r}\left(\mathbf{A}_{r+1}\right) \geq \sigma_{r}\left(\mathbf{A}_{r}\right) \geq \sigma_{r+1}\left(\mathbf{A}_{r+1}\right) .
$$

This interlacing property will prove useful for our purpose even though matrix A loses rows and not columns. Recall now that matrix $\mathbf{A}$ is of size $N \times N$. If we write

$$
A=\left(\begin{array}{l}
\mathbf{A}_{1} \\
\mathbf{A}_{2}
\end{array}\right)
$$

with both block matrices of size $K \times N$, and

$$
\boldsymbol{\Phi} \mathbf{A}=\left(\begin{array}{c}
\mathbf{A}_{1} \\
0
\end{array}\right)
$$




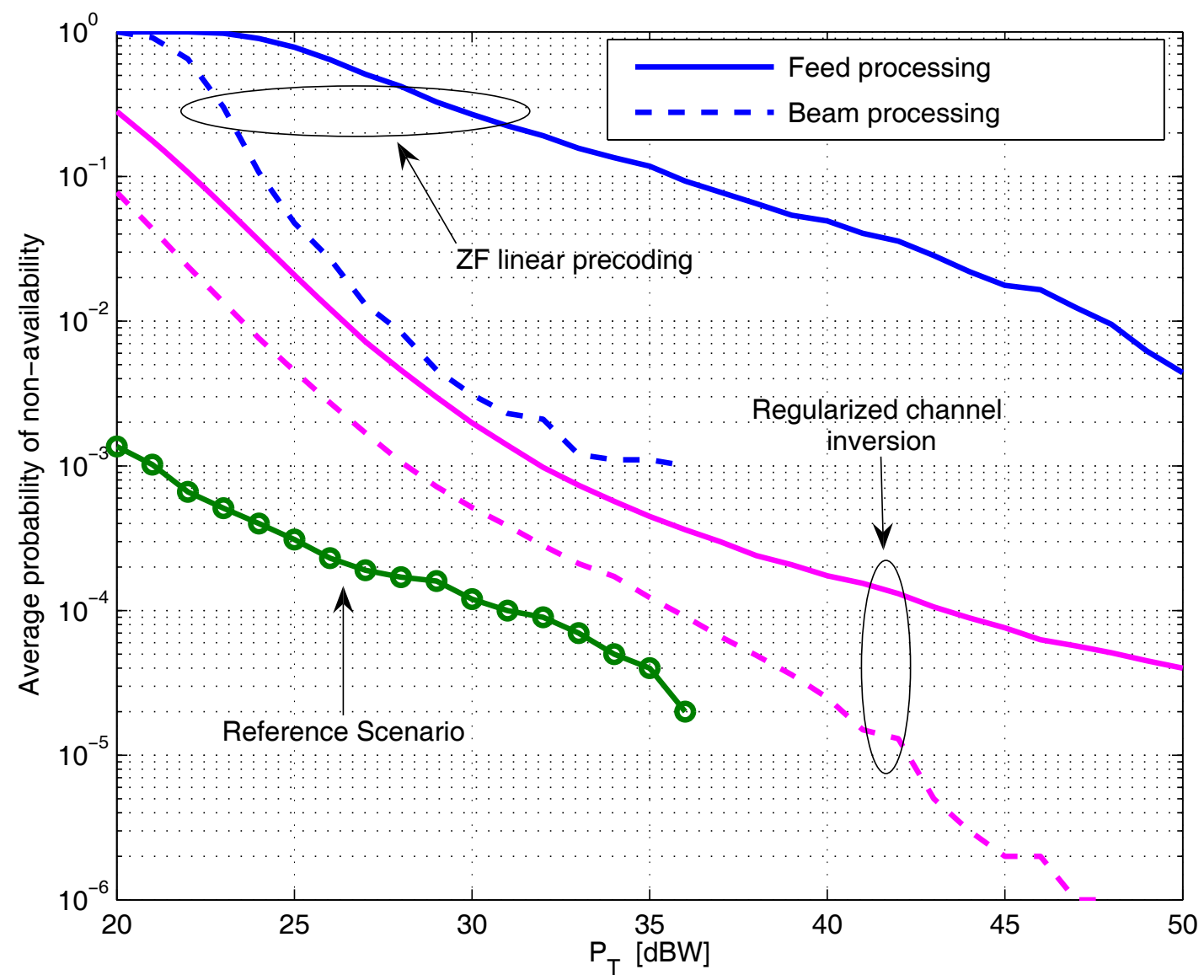

Figure 9 Regularized channel inversion and ZF linear precoding in beam and feed space: availability comparison with perfect CSI as a function of $P_{T}$.

then it is possible to define

$$
\tilde{\mathbf{A}}=\left(\begin{array}{cc}
\mathbf{A}_{1}^{H} & \mathbf{A}_{2}^{H} \\
\mathbf{0} & \mathbf{0}
\end{array}\right)
$$

whose singular values take the form $\sigma(\tilde{\mathbf{A}})=\left[\sigma_{1}(\mathbf{A}) \sigma_{2}(\mathbf{A}) \ldots \sigma_{K}(\mathbf{A}) 00 \ldots 0\right]$. If we now remove columns from the right, the interlacing property tells us that

$$
\sigma_{i}\left(\left(\begin{array}{c}
\mathbf{A}_{1} \\
\mathbf{0}
\end{array}\right)\right) \leq \sigma_{i}(\tilde{\mathbf{A}})
$$

which implies that

$$
\sigma_{i}(\boldsymbol{\Phi} \mathbf{A}) \leq \sigma_{i}(\mathbf{A})
$$

and concludes the proof. $\square$

\section{Appendix 3: Proof of Theorem 2}

In this appendix, we prove that

$$
\text { trace }\left\{\left(\mathbf{H}^{H} \mathbf{H}\right)^{-1}\right\} \leq \operatorname{trace}\left\{\mathbf{B}^{H}\left(\mathbf{B} \mathbf{H} \mathbf{H}^{H} \mathbf{B}^{H}\right)^{-1} \mathbf{B}\right\} \text {. }
$$

With the following singular value decomposition $\mathbf{H}=$ $\mathbf{V} \Sigma_{H} \mathbf{U}^{H}$, the left-hand side in (42) can be simplified as

$$
\operatorname{trace}\left\{\left(\mathbf{H}^{H} \mathbf{H}\right)^{-1}\right\}=\sum_{k=1}^{K} \frac{1}{\sigma_{k}^{2}(\mathbf{H})}
$$

where $\sigma_{k}^{2}(\mathbf{H})$ denotes the $k$ th largest singular value of H.

Similarly, denoting $\mathbf{B}=\mathbf{W} \Sigma_{B} \mathbf{Q}^{H}$, the right-hand side in (42) can easily be worked out as

$$
\operatorname{trace}\left\{\mathbf{B}^{H}\left(\mathbf{B} \mathbf{H} \mathbf{H}^{H} \mathbf{B}^{H}\right)^{-1} \mathbf{B}\right\}=\operatorname{trace}\left\{\left(\mathbf{Z}_{1}^{H} \mathbf{Z}_{1}\right)^{-1}\left(\Sigma_{H}^{H} \Sigma_{H}\right)^{-1}\right\}
$$




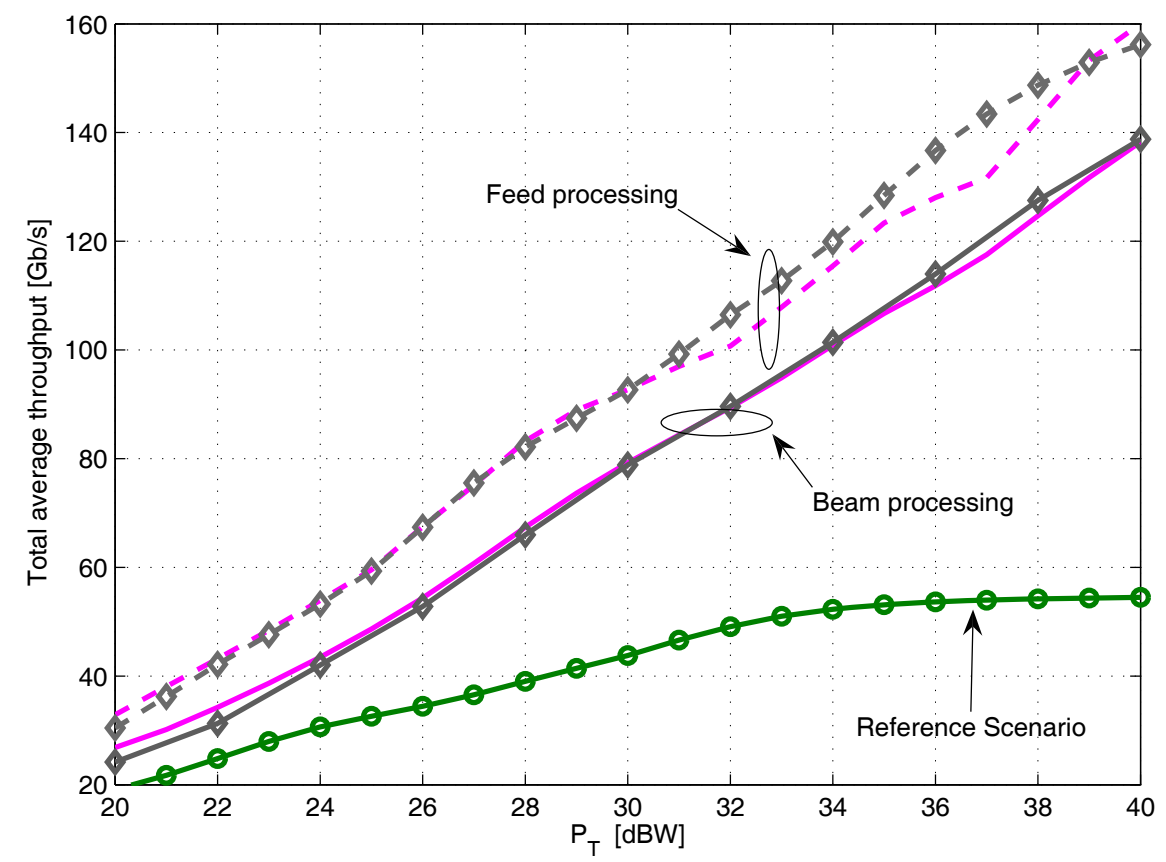

Figure 10 Throughput comparison with perfect CSI as a function of $P_{T}$. Legend: no marker-regularized channel inversion, $\mathbb{Q}-U p C o n s t$ MMSE precoder.

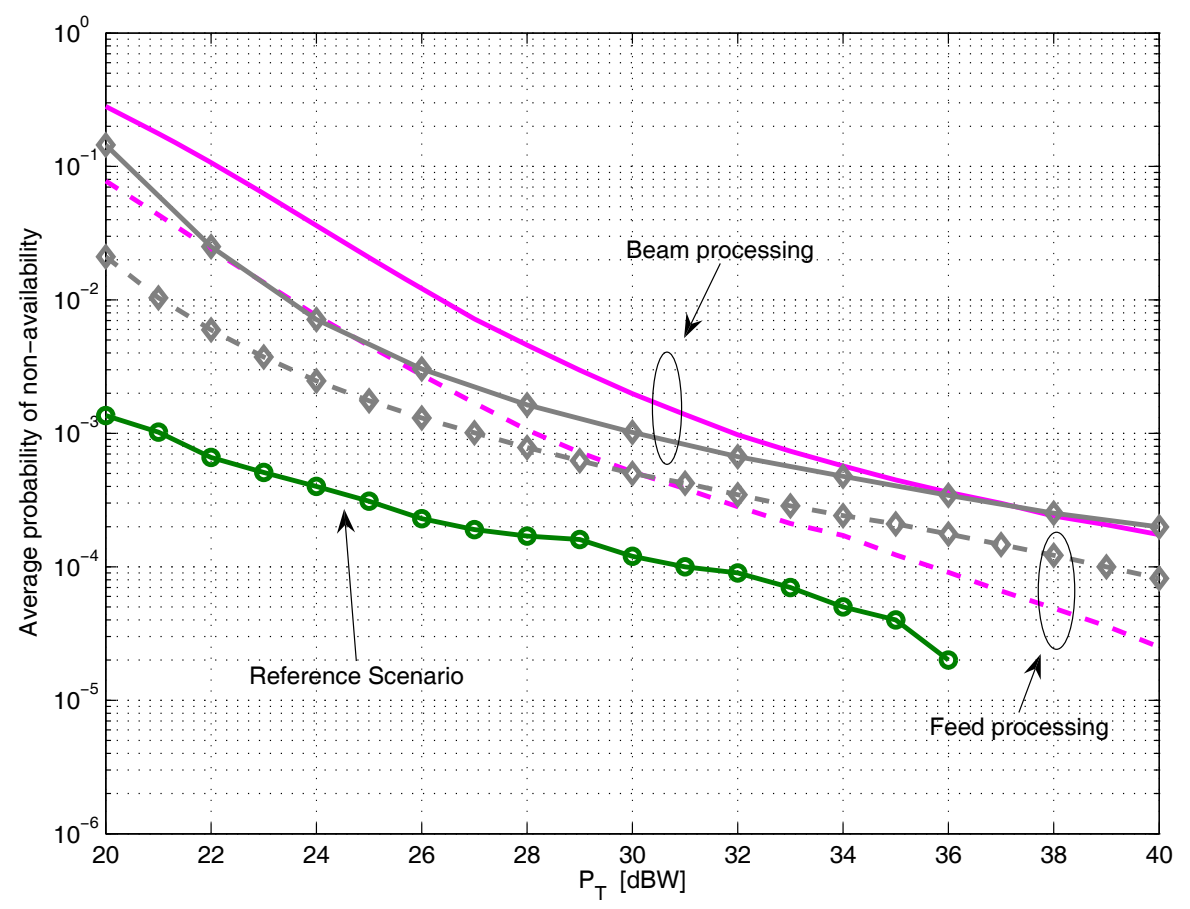

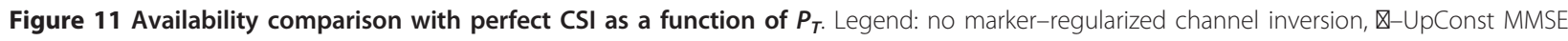
precoder. 


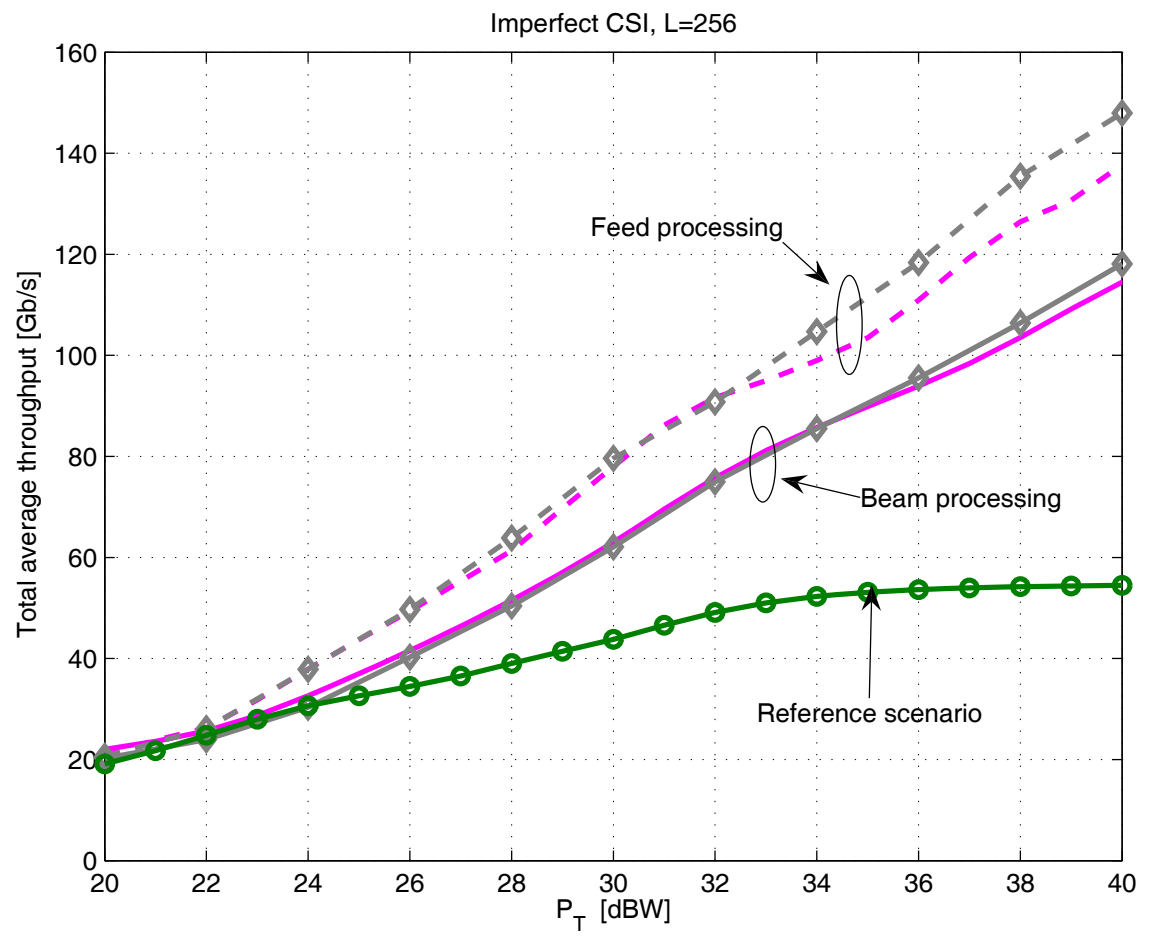

Figure 12 Throughput comparison with imperfect CSI as a function of $P_{T}(L=256)$. Legend: no marker-regularized channel inversion, Q-UpConst MMSE precoder.

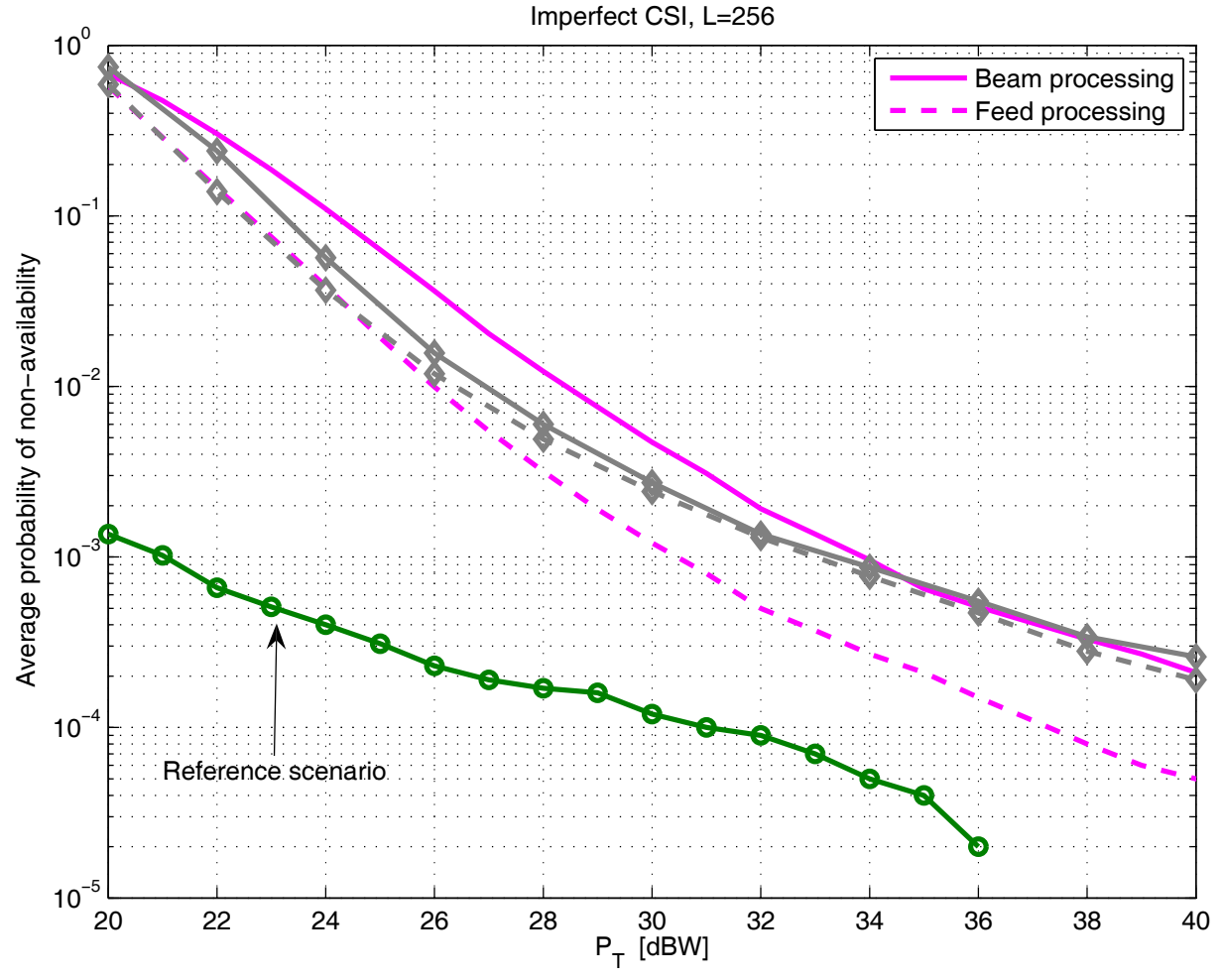

Figure 13 Availability comparison with imperfect CSI as a function of $P_{T}(L=256)$. Legend: no marker-regularized channel inversion, \-UpConst MMSE precoder. 


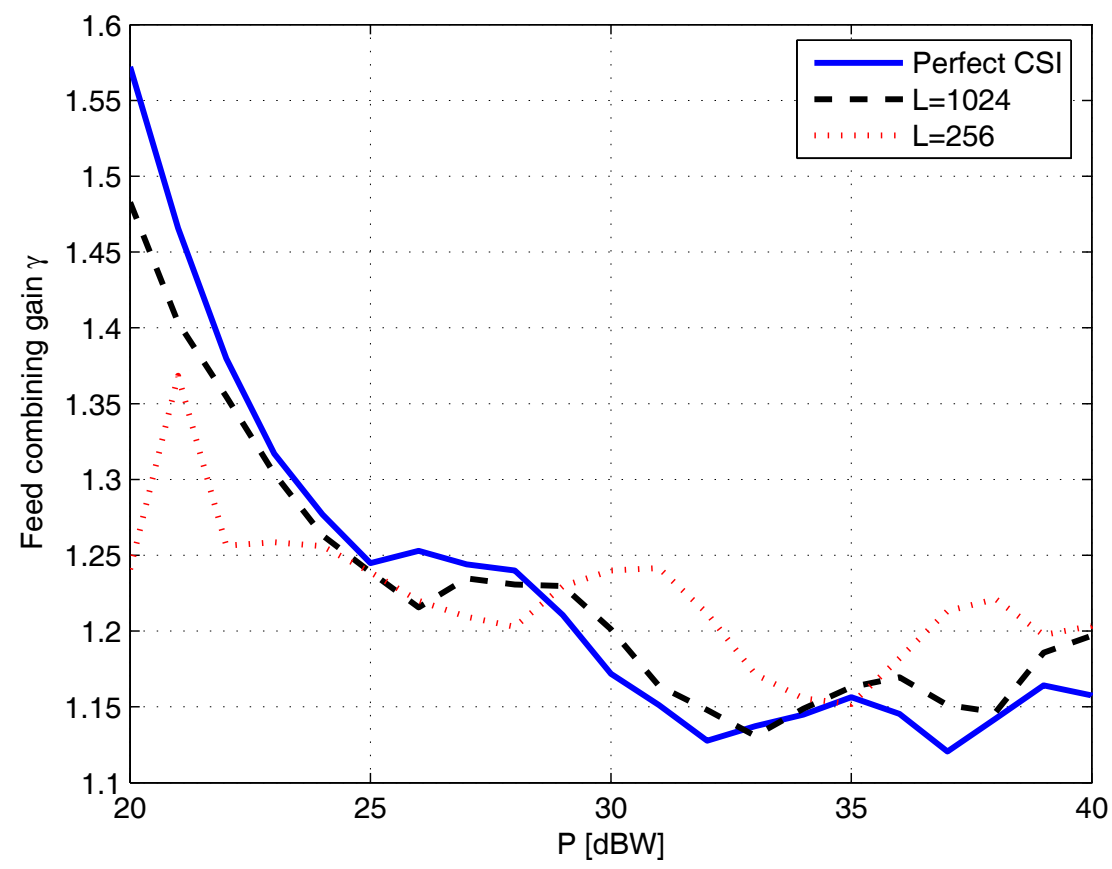

Figure 14 Feed combining gain $\gamma$ for the forward link as a function of the total transmit power $P_{T}$, for different degrees of CSI.

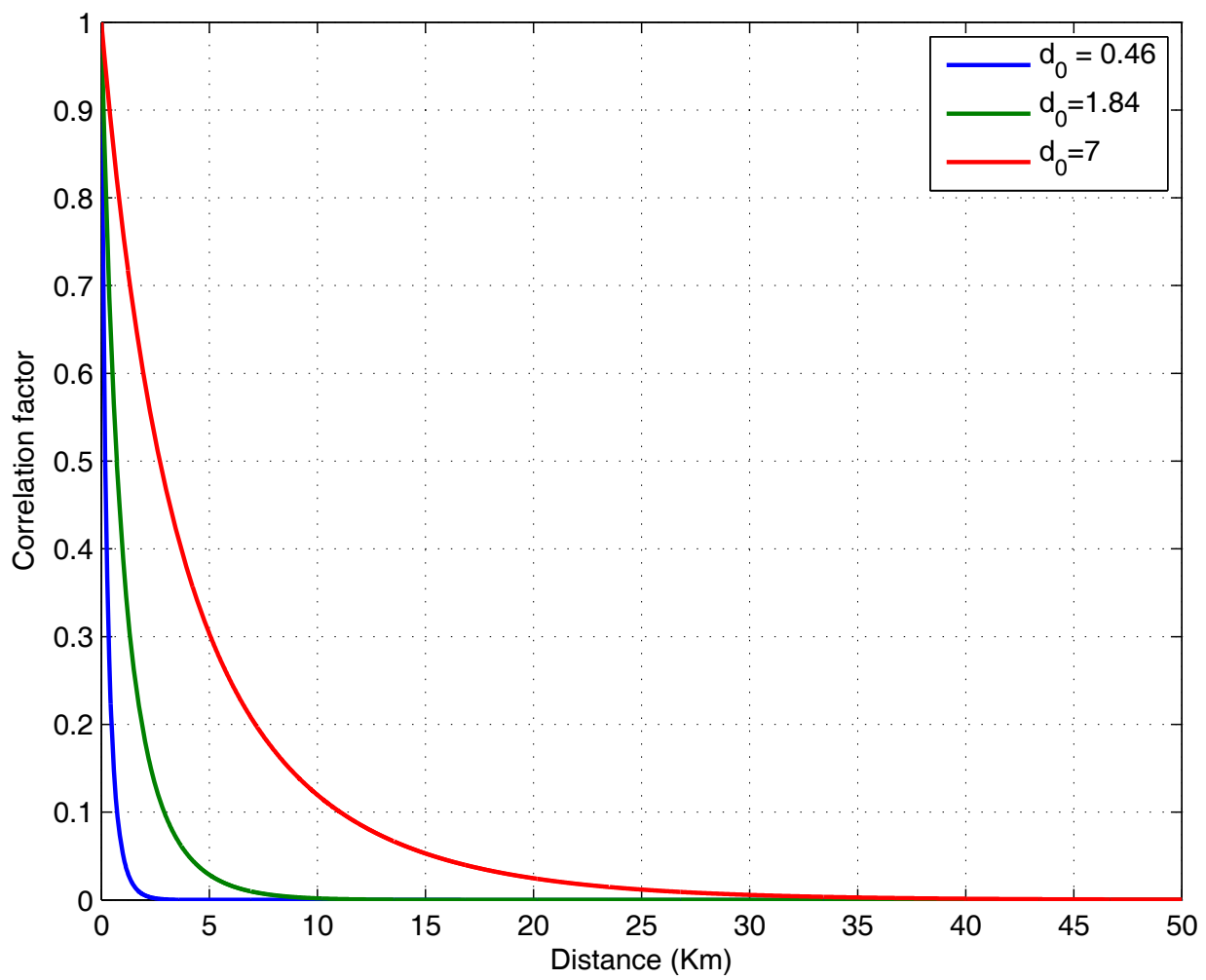

Figure 15 Evolution of the correlation factor with respect to the distance between the two points, natural units 


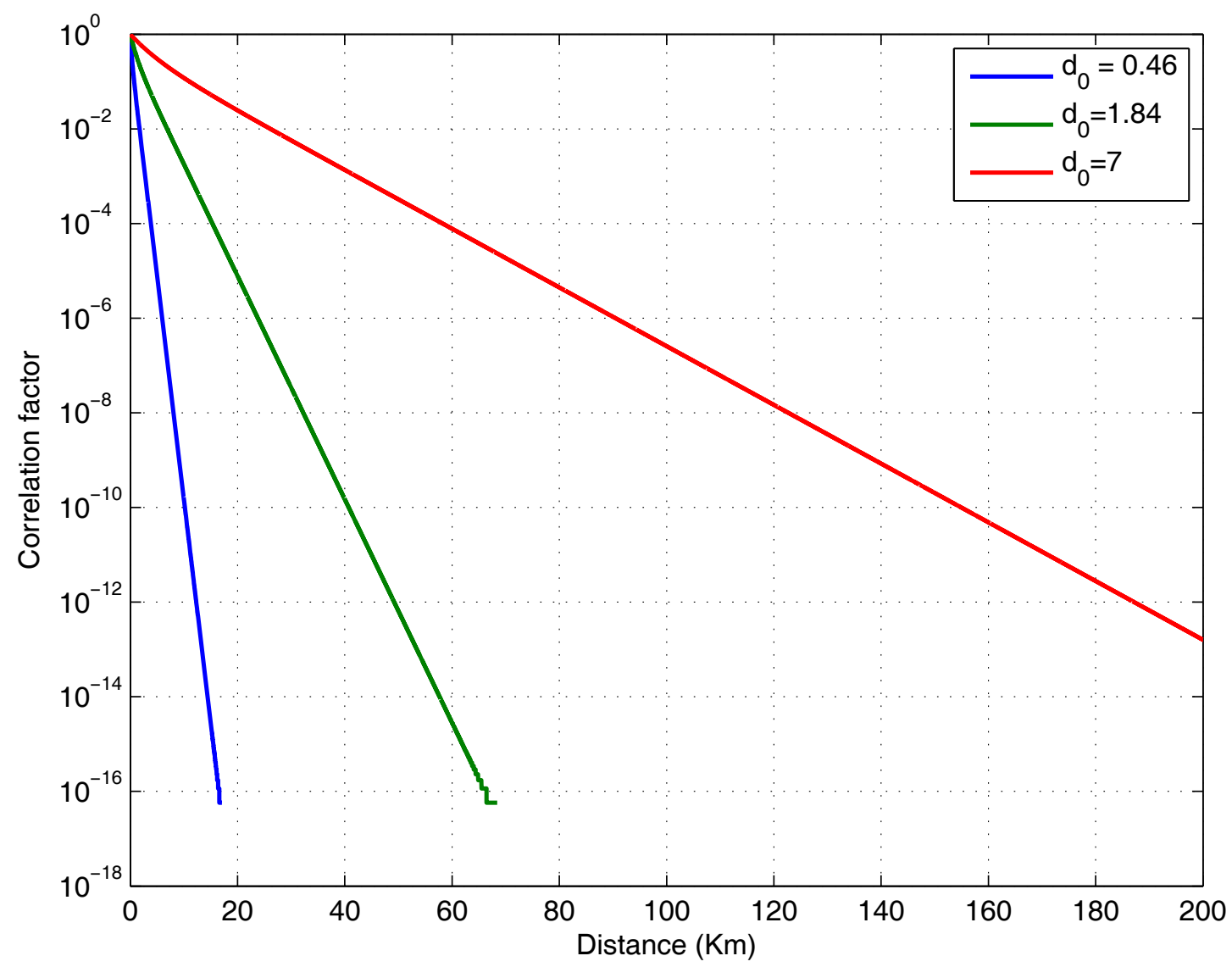

Figure 16 Evolution of the correlation factor with respect to the distance between the two points, logarithmic scale.

with the following definitions

$$
\begin{aligned}
& \mathbf{Z} \doteq \mathbf{Q}^{H} \mathbf{V} \\
& \doteq\left(\begin{array}{ll}
\mathbf{Z}_{1} & \mathbf{Z}_{2} \\
\mathbf{Z}_{3} & \mathbf{Z}_{4}
\end{array}\right)
\end{aligned}
$$

where the submatrix $\mathbf{Z}_{1}$ is of size $K \times K$, submatrices $\mathbf{Z}_{2}$ and $\mathbf{Z}_{3}^{H}$ are $K \times(N-K)$, and submatrix $\mathbf{Z}_{4}$ is $(N-K)$ $\times(N-K)$. We denote the $k$ th largest eigenvalue of $\mathbf{Z}_{1}^{H} \mathbf{Z}_{1}$ by $\lambda_{k}\left(\mathbf{Z}_{1}^{H} \mathbf{Z}_{1}\right)$. Let us first realize that

$$
\lambda_{k}\left(\mathbf{Z}_{1}^{H} \mathbf{Z}_{1}\right) \leq 1, k=1, \ldots, K .
$$

Indeed, since $\mathbf{Z}$ is a unitary matrix, we have that $\mathbf{Z}^{H} \mathbf{Z}$ $=\mathbf{I}_{N}$, which implies that

$$
\mathbf{Z}_{1}^{H} \mathbf{Z}_{1}+\mathbf{Z}_{3}^{H} \mathbf{Z}_{3}=\mathbf{I}_{K}
$$

With the following eigenvalue decomposition $\mathbf{Z}_{1}^{H} \mathbf{Z}_{1}=\mathbf{M} \operatorname{diag}\left(\lambda_{1}\left(\mathbf{Z}_{1}^{H} \mathbf{Z}_{1}\right), \ldots, \lambda_{K}\left(\mathbf{Z}_{1}^{H} \mathbf{Z}_{1}\right)\right) \mathbf{M}^{H}$, we can rewrite (48) as

$$
\operatorname{diag}\left(\lambda_{1}\left(\mathbf{Z}_{1}^{H} \mathbf{Z}_{1}\right), \ldots, \lambda_{K}\left(\mathbf{Z}_{1}^{H} \mathbf{Z}_{1}\right)\right)=\mathbf{I}_{K}-\mathbf{M}^{H} \mathbf{Z}_{3}^{H} \mathbf{Z}_{3}(\mathbf{M} 9)
$$

Hence, the matrix $\mathbf{M}^{H} \mathbf{Z}_{3}^{H} \mathbf{Z}_{3} \mathbf{M}$ has to be diagonal. Moreover, it has to have positive elements on the diagonal since $\mathbf{Z}_{3}^{H} \mathbf{Z}_{3}$ is semi positive definite, which proves (47).

Finally, by Theorem H.1.h in [30], we have that

$$
\operatorname{trace}\left\{\left(\mathbf{Z}_{1}^{H} \mathbf{Z}_{1}\right)^{-1}\left(\Sigma_{H}^{H} \sum_{H}\right)^{-1}\right\} \geq \sum_{k=1}^{K} \frac{1}{\lambda_{K-k+1}\left(\mathbf{Z}_{1}^{H} \mathbf{Z}_{1}\right)} \frac{1}{\sigma_{k}^{2}(\mathbf{H})}
$$

$$
\geq \sum_{k=1}^{K} \frac{1}{\sigma_{k}^{2}(\mathbf{H})}
$$


where (51) follows from (47), and concludes the proof. Note that the inequality becomes an equality if $\mathbf{Z}=\mathbf{I}_{N}$ , that is, $\mathbf{Q}^{H} \mathbf{V}=\mathbf{I}_{N}$. In others words, the equality is reached if $\mathbf{H}$ and $\mathbf{B}$ share the same left and right singular vectors, respectively. $\square$

\section{Acknowledgements}

The authors would like to acknowledge the work of the University of Bologna and Technical University of Graz teams, in particular, M. Bergmann, W. Gappmair, E. A. Candreva, G.E. Corazza, F. Lombardo, I. Thibault, and A. VanelliCoralli. We are also in debt with Riccardo de Gaudenzi for his valuable comments. Research supported by ESA contract 23089/10/NL/CLP "SatNEx Network of Experts", the European Regional Development Fund (ERDF) and the Spanish Government under projects DYNACS (TEC2010-21245-C02-02/TCM) and COMONSENS (CONSOLIDER-INGENIO 2010 CSD2008-00010), and the Galician Regional Government under projects "Consolidation of Research Units" 2009/62 and 2010/85. The work of B. Devillers was also partially supported by the Spanish Government under project TEC2010-17816 (JUNTOS). The work of A. Pérez-Neira had been supported by the Spanish Government under project TEC2008-06327-C03-01 and the Catalan Government under the grant 22009SGR0891. Some preliminary results of this study were presented at Asilomar and Globecom 2011 conferences.

\section{Author details}

'Signal Theory and Communications Department, University of Vigo, 36310 Vigo, Spain ${ }^{2}$ Centre Tecnològic de Telecomunicacions de Catalunya (CTTC), 08860 Castelldefels, Barcelona, Spain ${ }^{3}$ Department of Signal Processing and Communications, Universitat Politècnica de Catalunya, 08034 Barcelona, Spain

\section{Competing interests}

The authors declare that they have no competing interests.

\section{Received: 15 November 2011 Accepted: 5 April 2012}

Published: 5 April 2012

\section{References}

1. D Brandel, W Watson, A Weinberg, Nasa's advanced tracking and data relay satellite system for the years 2000 and beyond. Proc IEEE. 78, 1141-1151 (1990). doi:10.1109/5.56928

2. G Caire, M Debbah, L Cottatellucci, R De Gaudenzi, R Rinaldo, R Mueller, G Gallinaro, Perspectives of adopting interference mitigation techniques in the context of broadband multimedia satellite systems, in ICSSC 2005, 23rd AIAA International Communications Satellite Systems Conference, Rome, Italy. 1, 1-5 (September 2005)

3. L Cottatellucci, M Debbah, G Gallinaro, R Mueller, M Neri, R Rinaldo, Interference mitigation techniques for broadband satellite systems, in Proc 24th AIAA Int Commun Satell Systems Conf, ICSSC, San Diego CA. 1, 1-13 (2006)

4. P Angeletti, N Alagha, Space/ground beamforming techniques for emerging hybrid satellite terrestrial networks, in 27th International Communications Satellite Systems Conference (ICSSC 2009), Edimburgh, UK. 1, 1-6 (June 2009)

5. S Vishwanath, N Jindal, A Goldsmith, Duality, achievable rates, and sum-rate capacity of gaussian mimo broadcast channels. IEEE Trans Inf Theory. 49, 2658-2668 (2003). doi:10.1109/TIT.2003.817421

6. O Somekh, S Shamai, Shannon-theoretic approach to a gaussian cellular multiple-access channel with fading. IEEE Trans Inf Theory. 46, 1401-1425 (2000). doi:10.1109/18.850679

7. G Caire, S Shitz, On the achievable throughput of a multi-antenna gaussian broadcast channel. IEEE Trans Inf Theory. 49, 1691-1707 (2003). doi:10.1109/ TIT.2003.813523

8. T Yoo, A Goldsmith, On the optimality of multiantenna broadcast scheduling using zero-forcing beamforming. IEEE J Sel Areas Commun. 24, 528-541 (2006)

9. C Peel, B Hochwald, A Swindlehurst, A vector-perturbation technique for near-capacity multiantenna multiuser communication. Part I: channel inversion and regularization. IEEE Trans Commun. 53, 195-202 (2005). doi:10.1109/TCOMM.2004.840638
10. D Tse, P Viswanath, Fundamentals of Wireless Communication (Cambridge University Press, New York, NY, USA, 2005)

11. J Choi, Optimal Combining and Detection: Statistical Signal Processing for Communications, 1st edn. (Cambridge University Press, New York, NY, USA, 2010)

12. A Paulraj, R Nabar, D Gore, Introduction to Space-Time Wireless Communications, 1st edn. (Cambridge University Press, New York, NY, USA, 2008)

13. G Gallinaro, M Debbah, R Müller, R Rinaldo, A Vernucci, Interference mitigation for the reverse-link of interactive satellite networks, in 9th International Workshop on Signal Processing for Space Communications, Noordwijk, The Netherlands. 1, 1-7 (2006)

14. J Arnau-Yanez, M Bergmann, E Candreva, G Corazza, R de Gaudenzi, B Devillers, W Gappmair, F Lombardo, C Mosquera, A Perez-Neira, I Thibault, A Vanelli-Coralli, Hybrid space-ground processing for high-capacity multibeam satellite systems, in IEEE Global Telecommunications Conference (GLOBECOM 2011), Houston TX. 1, 1-6 (2011)

15. B Devillers, A Pérez-Neira, C Mosquera, Joint linear precoding and beamforming for the forward link of multi-beam broadband satellite systems, in Proc IEEE Global Communications Conference, GLOBECOM, Houston, Texas. 1, 1-6 (2011)

16. N Zorba, M Realp, A Perez-Neira, An improved partial CSIT random beamforming for multibeam satellite systems, in 10th International Workshop on Signal Processing for Space Communications, 2008 SPSC, Rhodes Island, Greece. 1, 1-8 (2008)

17. KP Liolis, AD Panagopoulos, PG Cottis, Multi-satellite MIMO communications at ku-band and above: investigations on spatial multiplexing for capacity improvement and selection diversity for interference mitigation. EURASIP J Wirel Commun Netw. 2007, 16-16 (2007)

18. A Ishimaru, J Ritcey, S Jaruwatanadilok, Y Kuga, A MIMO propagation channel model in a random medium. IEEE Trans Antennas and Propagation. 58, 178-186 (2010)

19. M Castro, G Granados, Cross-layer packet scheduler design of a multibeam broadband satellite system with adaptive coding and modulation. IEEE Trans Wirel Commun. 6, 248-258 (2007)

20. S Chatzinotas, G Zheng, B Ottersten, Joint precoding with flexible power constraints in multibeam satellite systems, in IEEE Global Telecommunications Conference (GLOBECOM), Houston TX. 1, 1-5 (2011)

21. SM Kay, Fundamentals of Statistical Signal Processing: Estimation Theory, (Prentice-Hall, Inc., Upper Saddle River, NJ, USA, 1993)

22. DVB.org, Digital Video Broadcasting (DVB); Second Generation DVB Interactive Satellite System; Part 2: Lower Layers for Satellite standard (2011)

23. H Brandt, O Lücke, V Boussemart, C Párraga-Niebla, T Flo, C Kissling, R Schweikert, Resources Management using adaptive fade mitigation techniques in DVB-S2/RCS multi-beam systems, in Proccedings of the 25th International Communications Satellite Systems Conference (ICSSC 2007), Seoul, South Korea. 1, 1-13 (2007)

24. P Viswanath, DNC Tse, Sum capacity of the vector Gaussian broadcast channel and uplink-downlink duality. IEEE Trans Inf Theory. 49, 1912-1921 (2003). doi:10.1109/TIT.2003.814483

25. B Gremont, M Filip, Simulation of a high frequency satellite link with a fade countermeasure, in IEE National Conference on Antennas and Propagation, York, UK. 1, 164-168 (1999)

26. GJ Ciach, WF Krajewski, Analysis and modeling of spatial correlation structure in small-scale rainfall in central oklahoma. Adv Water Resour. 29(10), 1450-1463 (2006). doi:10.1016/j.advwatres.2005.11.003

27. P-DM Arapoglou, E Kartsakli, GE Chatzarakis, PG Cottis, Cell-site diversity performance of Imds systems operating in heavy rain climatic regions. Int J Infrared Millimeter Waves. 25, 1345-1359 (2004). doi:10.1023/B: IJIM.0000045144.01224.bf

28. M Cheffena, L Braten, T Ekman, On the space-time variations of rain attenuation. IEEE Trans Antennas Propag. 57, 1771-1782 (2009)

29. GH Golub, CF Van Loan, Matrix Computations (Johns Hopkins Studies in Mathematical Sciences), 3rd edn. (The Johns Hopkins University Press, Baltimore, MD, 1996)

30. AW Marshall, I Olkin, Inequalities: Theory of Majorization and its Applications, (Academic Press, New York, NY, 1979)

doi:10.1186/1687-1499-2012-132

Cite this article as: Arnau et al:: Performance study of multiuser interference mitigation schemes for hybrid broadband multibeam satellite architectures. EURASIP Journal on Wireless Communications and Networking 2012 2012:132. 